\title{
Conservation and divergence of DNA replication control in Chlamydomonas reinhardtii
}

Running title: Analysis of DNA replication proteins in Chlamydomonas reinhardtii

Amy E. Ikui ${ }^{1 *}$, Noriko Ueki ${ }^{1}$, Kresti Pecani ${ }^{2}$, Frederick Cross ${ }^{2} *$

${ }^{1}$ Department of Biology, Brooklyn College, The City University of New York, Brooklyn NY

${ }^{2}$ Laboratory of Cell Cycle Genetics, The Rockefeller University, New York, NY

* To whom correspondence should be addressed 


\begin{abstract}
We recently isolated temperature-sensitive cell cycle mutants in Chlamydomonas reinhardtii for which the causative mutations were located in genes annotated for potential involvement in DNA replication. Chlamydomonas has a very long G1 period during which cells grow up to $\sim 10$-fold without division, followed by rapid cycles of DNA replication and mitosis ('multiple fission'). All of the candidate DNA replication mutants tested were defective in completion of the first round of DNA replication, and also failed to produce mitotic spindles. For a subset of the mutants, we rescued temperature-sensitive lethality with tagged transgenes and used the resulting strains to analyze abundance and localization control of the tagged protein. All of the DNA replication proteins tested were essentially undetectable until late G1, accumulated during the period of multiple fission and then were degraded as cells completed their terminal divisions. MCM4 and MCM6 were localized to the nucleus during the division cycle except for transient cytoplasmic localization during mitosis. CDC45 showed strict protein location to the nucleus and co-localized to spindles during mitosis. In contrast, CDC6 was detected in the nucleus only transiently during early divisions within the overall multiple fission cycle. Cdc6 protein levels were very low, but increased upon treatment with MG132, a proteasome inhibitor. We also tested if these DNA replication proteins are regulated by cyclin dependent kinase (CDK). There are two main CDKs in Chlamydomonas, CDKA1 and CDKB1. We found that CDC6 protein level was severely reduced in a $c d k a 1$ mutant, but not in a $c d k b 1$ mutant. MG132 did not detectably increase CDC6 levels in the cdkal mutant, suggesting that CDKA1 upregulates CDC6 at the transcription level. Since MCM4, MCM6 and CDC6 were all essentially undetectable during the long G1 before DNA replication cycles began, we speculate that loading of origins with the MCM helicase may not occur until the end of the long G1, unlike in the budding yeast system. These results provide a microbial framework for approaching replication control in the plant kingdom.
\end{abstract}




\section{INTRODUCTION}

The cell cycle is an ordered set of cellular processes in which DNA is replicated and segregated into two identical daughter cells. In eukaryotes, the cell cycle is controlled by the cyclin dependent kinase, CDK, coupled with cyclins [1]. Chlamydomonas reinhardtii is a single cell green algae which divides by 'multiple fission': a long G1 phase with massive cell growth, followed by typically 3-4 rounds of S/M phase that consists of DNA replication, chromosome segregation and cytokinesis [2]. The resulting daughter cells remain within the mother cell wall, finally hatching to produce 8-16 small newborn G1 cells that restart the cycle. There are two major CDKs in Chlamydomonas reinhardtii, $C D K A 1$ (a homologue of animal $C D K 1 / C D C 2$ ) and $C D K B 1$ (a $C D K$ specific to the plant kingdom) [3, 4]. While $C D K 1$ is the direct trigger of mitosis in yeast and animals, $C D K A 1$ in the plant kingdom specifically functions early in the cell cycle; mitosis is promoted by the plant-kingdom-specific $C D K B 1$. One important role of $C D K A 1$ early in the cell cycle is transcriptional activation of a large battery of genes required for cell division that are turned on shortly before the division cycles begin $[5,6]$.

DNA replication has been studied extensively in the budding yeast $S$. cerevisiae. To initiate DNA replication in yeast, ORC (ㅁigin recognition complex) binds to origins followed by Cdc6 (ㅌell division cycle), Cdt1p and Mcm2-7 (Minichromosome maintenance), forming the pre-replicative complex (pre-RC) [7-10]. CDK activity promotes export of the MCM complex from the nucleus, so that Mcm2-7 loading on chromatin is restricted to late $\mathrm{M}$ and early $\mathrm{G1}$ phase when CDK activity is inhibited [11]. Firing of MCM-loaded ('licensed') replication origins, by contrast, requires CDK activity to phosphorylate multiple proteins to assemble a functional replication complex [12]. Once DNA replication is initiated, the pre-RC components such as Cdc6, Mcm2-7, and the ORC complex are phosphorylated by Cyclin/CDK; these multiple mechanisms prevent a second round of MCM reloading and consequent DNA replication from occurring before mitosis [13]. For example, Cdc6 is phosphorylated by Cyclin/CDK, targeting it for ubiquitin-mediated proteolysis through the SCF complex [14-17], Mcm2-7 is exported from the nucleus as noted above, and ORC function is inhibited by phosphorylation. 
These mechanisms collectively prevent origin relicensing within $\mathrm{S}$ phase.

The pre-RC proteins are mostly conserved in humans, but there are significant functional differences. While ScOrc1-6 binds to specific origin DNA sequences throughout the cell cycle, HsOrc1-6 is transiently associated with chromatin during G1 phase, and no origin consensus sequence has been defined [18]. HsMcm2 and HsMcm4 are phosphorylated by Cyclin B/Cdc2 during the G2-M phase [19]. Phosphorylation inhibits HsMcm2-7 reloading onto chromatin [20], but does not cause nuclear export as in yeast. Unlike in yeast, HsCdc6 is localized to the nucleus in G1 with a small fraction of HsCdc6 in the cytoplasm during S phase [21]. SCF-dependent HsCdc6 degradation inhibits DNA re-replication [22]. In contrast to yeast, where CDK-dependent phosphorylation promotes Cdc6 proteolysis, CDK-dependent HsCdc6 phosphorylation prevents Cdc6 proteolysis [23, 24].

After origin licensing, the pre-loading complex (pre-LC) is formed and activated by CDK and DDK kinases. Several critical proteins of the pre-LC in yeast are poorly conserved in vertebrates, such as Sld2. CDK-dependent ScSld2 phosphorylation is required for initiation of DNA replication [25, 26]. RecQL4 in vertebrates shows a unique Nterminal domain with limited homology to ScSld2, and a large C-terminal domain with 3'5' DNA helicase activity. However, only the N-terminal region of RecQL4, lacking helicase activity and CDK phosphorylation sites, is required to trigger vertebrate DNA replication [39]. In yeast, Dpb11 is a scaffold protein used to recruit Sld2 and Sld3 through CDK dependent phosphorylation in order to assemble the Cdc45-Mcm-GINS (CMG complex). The human homologue of Dpb11, TopBP1, directly recruits Cdc45 onto replication origins [27]. Overall, the pre-LC is diverse both at the level of sequence and function between yeast and humans in contrast to the tight conservation of pre-RC across eukaryotes.

ORC, CDC6 and CDT1 have been identified in the land plant Arabidopsis thaliana [28]. AtORC1b is expressed only in proliferating cells. AtCDC6 is expressed during Sphase under control of E2F dependent transcription [29]. Ectopic expression of AtCDC6 or AtCDT1 induces endoreplication with altered ploidy levels [30]. AtCDC6 is degraded in a cell-free system in a proteasome-dependent manner [30]. AtMCM2-7 was expressed 
preferentially in tissues where DNA replication was occurring [31]. While AtMCM7 is transiently localized to the nucleus during G1 [32], AtMCM5 and AtMCM7 localize to the nucleus in G1, S and G2 phases and then translocate to the cytoplasm during mitosis [31]. MCM6 from maize is expressed at its highest level in the nucleus during G1 and then translocates to the cytoplasm during S phase [33]. Therefore, the function of MCM2-7 in plants may be regulated by localization. Plant $O R C$ and $C D C 6$ have been shown genetically to be essential; however, the absence of conditional mutant alleles, and the lack of a simple cell cycle assay system in plants, has made it difficult to functionally address mechanisms of DNA replication control in the plant kingdom [34].

In this study, we analyzed DNA replication mutants identified in our previous UVmutagenesis screening in Chlamydomonas reinhardtii $[35,36]$, concentrating on pre-RC components and their relationship to CDKA1 and CDKB1 activity.

\section{RESULTS}

\section{Completion of the first cycle of DNA replication and nuclear division are inhibited in DNA replication mutants}

In order to monitor cell cycle progression and to obtain a synchronous cell population in Chlamydomonas reinhardtii, we studied cells synchronized by nitrogen deprivation (causing arrest in G1) and refeeding. At intervals, cells were collected, fixed and stained with Sytox followed by flow cytometry analysis (FACS). By 12-14 hrs after release, wild type cells undergo multiple synchronous cycles of DNA replication and mitosis within the mother cell wall, yielding multinucleate cells producing signals of DNA content of 1, 2, 4, 8 and 16C (Figure 1A and 1B). Chloroplast DNA replication occurs early and uncoupled from the nuclear replication cycle and likely only accounts for a small part of the total FACS signal [37].

The DNA replication mutant cells showed varying degrees of defect. Some showed no detectable increase in DNA content; others appeared to complete most of the first DNA replication cycle. However, all mutants were severely defective in nuclear division and entry into a second round of DNA replication (Figure 1A and 1B). Thus DNA replication is 
inhibited in the temperature sensitive (ts) DNA replication mutants as expected; in addition, incomplete DNA replication in the first cycle may block both nuclear division and reinitiation for a second round. Lack of nuclear division correlated with absence of detectable mitotic spindle formation by anti-tubulin immunofluorescence (Figure 1C). In yeast and animals, incomplete replication similarly triggers efficient blocks to mitotic progression [38].

\section{Amino acid sequence alignment of DNA replication proteins}

Sequence alignments between the Chlamydomonas genes tested here generally showed the highest homology to land plant (Arabidopsis) genes, but also a strong alignment to animal and fungal sequences (Table 1). Interestingly, in many cases, alignment between Chlamydomonas and animal sequences was stronger than between

Chlamydomonas and fungal sequences, most likely due to accelerated sequence evolution in fungal lineages. These alignments allow confident assignment of the Chlamydomonas mutants to the indicated orthologous animal sequences (Table 1).

The C-terminal region of Cre10.g455600 shared high-scoring alignment with MmOrc1 and also shared lesser homology with AtCDC6 and MmCdc6, confirming the similarity between Orc1 and Cdc6 [39]. ORC1 contains an ATPase associated with diverse cellular Activities (AAA+) domain, and the div74-1 mutation was located in this domain (Figure 2A). Similar to MmOrc1, Cre10.g455600 also contains a PHD (Plant

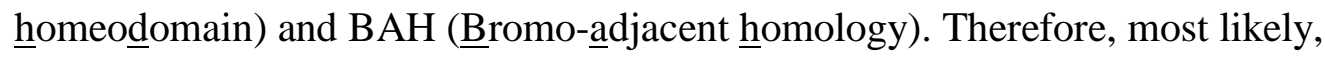
Cre10.g455600 is the ORC1 homologue in Chlamydomonas.

As noted above, the functional equivalent of budding yeast Sld 2 in animals is RecQL4. Homology between ScSld2 and animal RecQL4 is almost undetectable, at most, restricted to a small N-terminal region; however, this region, and not the large RecQL4 helicase domain, is required for DNA replication in Xenopus [40]. Chlamydomonas DIV20 is a RecQL4 ortholog [35] that also has weak but detectable N-terminal ScSld2 homology (Table 1; Figure 2C). Surprisingly, though, mutations of two independent mutants that inactivate DIV20 for DNA replication, map adjacent to the helicase domain in the C- 
terminal extension specific to the RecQ4 family. This region is dispensable for replication in Xenopus (Figure 2C) [40].

The RecQ family comprises a large number of proteins in Arabidopsis and other land plants. If our assignment of DIV2O as a functional homolog of animal RecQL4 is correct, then we suggest that the Arabidopsis equivalent replication protein is AT1G27880 (Figure. 2C). Even though AT1G27880 is annotated (https://phytozome.jgi.doe.gov/) as a 'DEAD/DEAH RNA helicase', it is a protein with similar overall structure to DIV20/Cre15.g634701. Similarly, if DIV21/Cre12.g515900 aligns functionally to animal TopBP1 for replication, then a likely Arabidopsis homolog might be AT1G77320, a BRCT-repeat-containing protein like TopBP1 and Chlamydomonas DIV21 (Figure 2B). The functional significance of the BRCT repeats in DIV21 is emphasized by the finding that two independent ts-lethal div21 mutations lie in the region of one of its BRCT repeats. These examples point to the utility of functional identification of genes in the Chlamydomonas genome (mainly single-copy) to help navigate the more complex, geneduplicate-rich land plant genomes.

\section{Synthetic lethality between pre-RC mutants}

If Chlamydomonas pre-RC components function in complexes, as do their yeast and human orthologs, then strong genetic interactions might be expected between mutations in different genes in this set. We inter-crossed pre-RC mutants orc1, cdc6, mcm4, mcm6$981 \mathrm{~A}$ and $\mathrm{mcm6}-\mathrm{GHI}[36]$ and analyzed viability of double mutant progeny at the permissive temperature. $m c m 6-981 A$ was synthetically lethal with orc $1, c d c 6$ and $m c m 4$ (Figure 3A). mcm6-GHI, showed mild synthetic lethality with orc1, though it was not lethal at the permissive temperature when combined with $c d c 6$ (Figures 3B). The orcl cdc6 double mutant was also mildly sick (Figure 3C). The genetic interaction was summarized in Figure 3D. Frequent synthetic lethality in this mutant set is consistent with the proteins that form functional complexes, as expected from prior work in yeast (see Introduction).

\section{Transgenes expressing fluorescent fusion proteins rescued temperature sensitivity of}




\section{the replication mutants}

We created universal tagging plasmids containing mCherry, Venus or GFP to be used in Chlamydomonas. The plasmid backbone was generated from CDKB1 plasmid which contains mCherry-tagged $C D K B 1$ gene [41]. The final plasmids contain aphVIII $C D S$ for paramycin resistance that is useful for drug selection in Chlamydomonas, an Ampicillin resistant gene for bacterial selection, and mCherry, GFP or Venus followed by the $C D K B 1$ 3'UTR, as well as multiple cloning sites for insertions of desired promoters and coding sequences (Figure 4A).

We amplified CDC45 PCR product from genomic DNA and inserted it into to the mCherry plasmid through Gibson assembly. The resulting CDC45-mCherry plasmid was linearized with $\mathrm{XbaI}$ and electroporated into the $c d c 45$ ts mutants. Tranformants were selected for antibiotic resistance at $21^{\circ} \mathrm{C}$ (permissive temperature) and then screened at the non-permissive temperature, $33^{\circ} \mathrm{C}$ (Figure 4B). A small proportion of transformants grew at $33^{\circ} \mathrm{C}$; such low co-rescue is a general feature of Chlamydomonas transformation, probably resulting from frequent breakage or silencing of transforming DNA. Colonies that grew at $33^{\circ} \mathrm{C}$ were picked and their genomic DNA was tested by PCR for the presence of mCherry, the region covering the promoter and the ampicillin resistance gene. No temperatureresistant colonies were obtained with the empty vector (Figure 4B).

Similarly, we constructed tagging plasmids to rescue other ts-lethal mutants. Efficiency of rescue in the selected transformants was confirmed by serial dilution (Figure 5A). Selected transformants were further subjected to western blotting to analyze the expression and size of the fusion protein (Figure 5B). Clones with efficient rescue and protein expression were backcrossed with wild type at least once before use in further experiments (Figure 5, red numbers). Transgenes in Chlamydomonas integrate at random locations, which could affect expression efficiency. In addition, the transgene plasmid is often broken or rearranged in Chlamydomonas before it is integrated. The Western blot analysis and genetic rescue ensures an approximately full-length fusion protein, but in principle the promoter region could be disrupted causing less efficient transcription. In order to maximize the likelihood that the selected transformant would contain a complete 
transgene, we designed the primers to detect inclusion of the promoter, ampicillin resistant gene and inclusion of the tagged gene.

\section{Expression of DNA replication proteins during the division cycle}

All transgenes were constructed with their native promoters, so that we could analyze accumulation of MCM6-mCherry, CDC45-mCherry, ORC1-Venus and CDC6mCherry fusion proteins at endogenous levels throughout the cell cycle. We included a strain expressing CDKB1-mCherry as a positive control since it is known to accumulate specifically in division-phase cells [41]. Cells were arrested at G1 phase as described above, then released into complete media at $33^{\circ} \mathrm{C}$. Since the transgene was expressed in the context of the endogenous ts-lethal mutation, cell cycle progression was dependent on the fluorescent fusion protein in each case. CDKB1-mCherry was first expressed at $10 \mathrm{hrs}$, maximized around $14 \mathrm{hrs}$ and declined by $24 \mathrm{hrs}$ as previously shown [41]. These times correlate with the period of multiple fission cycles. Similarly, the maximum levels of tagged MCM6, ORC1, CDC6 and CDC45 were observed at 14 hrs after release (Figure 6A and 6B). ORC1 and CDC6 mCherry fusion protein levels were undetectable by direct Western analysis. Therefore these tagged proteins were pulled down using Dynabeads conjugated with mCherry nanobody to concentrate the protein before Western analysis. FACS analysis of CDC6-mCherry showed that DNA replication initiated at 12 hrs as expected in this procedure (Figure 6C). We conclude that pre-RC proteins are essentially absent during the long G1 phase in Chlamydomonas, and like CDKB1 are expressed only during the rapid divisions of the multiple fission cycle. This is an interesting contrast to the yeast system, in which pre-RC components are loaded onto origins starting in late mitosis and are bound throughout G1 phase.

\section{MCM proteins are localized to the nucleus during the division cycle with a transient release during mitosis}

Mcm6-mCherry protein localization was analyzed by indirectimmunofluorescence using anti-mCherry antibody. Mcm6 was localized to the nucleus in 
wild type cells, though sporadic cells with dispersed Mcm6 protein in the cytoplasm were detected (Figure 7A, white triangles). We used a $c d c 27$ cell cycle mutant in order to examine Mcm6 localization in metaphase-blocked cells. CDC27 is a part of the Anaphase Promoting Complex (APC) [42], required for the metaphase-anaphase transition [35, 41]. MCM6 was diffused from the nucleus in metaphase-arrested $c d c 27$ cells, and co-staining of microtubules showed that these cells contained mitotic spindles (Figure 7B). After $16 \mathrm{hrs}$ the cell cycle was completely arrested in G2/M phase after completion of a single round of DNA replication in the $c d c 27$ mutant (Figure 7B). Most likely, the sporadic wild type cells with diffuse MCM6-mCherry localization are cells transiting through metaphase.

In budding yeast, translocation of Mcm2-7 to the cytoplasm in S-phase after origin firing may prevent origin re-loading within a given S-phase [43]. Localization of Mcm2-7 is controlled by Cdk1-dependent phosphorylation [44, 45]. It is possible that transient mitotic loss of MCM6 from the nucleus in Chlamydomonas is regulated similarly. We tested if CDKA1 or CDKB1 controls MCM6 localization and found that MCM6 stayed in the nucleus in both $c d k a l$ and $c d k b 1$ mutants (Figure 7C), while leaving the nucleus in the $c d c 27$ background in which CDKA1 and CDKB1 activities are both high [48]. Thus it is possible that CDK activity regulates Mcm6 localization in Chlamydomonas as in yeast; we lack direct evidence for this idea, however. Western blotting analysis showed that the MCM6-mCherry protein was readily detectable in $r n r l, c d k b l$ and $c d c 27$ mutants similar to the level in wild type cells and was slightly suppressed in the cdkal mutant (Figure 7D).

To extend these findings to a different member of the MCM complex, we monitored MCM4-Venus localization in single cells, using a variant of procedures developed for budding yeast time-lapse microscopy [46, 47] (see Methods). Cells expressing MCM4-Venus were monitored at 3-min resolution, starting in G1 and extending to completion of a multiple fission cycle. MCM4-Venus was absent in the initially plated G1 cells, but began to accumulate approximately 2 hrs before the first division (Figure 8A) (Movie1). MCM4-Venus was localized to a small region in the cell anterior that we assume is the cell nucleus based on size, shape, position and similarity to the MCM6-mCherry nuclear localization in immunofluorescence experiments in Figure 7. The MCM4-Venus 
signal was greatly reduced from the nucleus in the frame right before the first division, but reappeared almost immediately after division (Figure 8A and 8C). Quantification of the total MCM4-Venus signal across the cell indicated that the reduction or loss of nuclear intensity occurred without significant change in total cellular levels. Therefore, we attribute the loss of nuclear signal to transient diffusion into the cytoplasm rather than to MCM4 proteolysis and rapid resynthesis. MCM4-Venus transient loss was restricted to a single 3min frame in almost all cells observed. We observed the same one-frame loss of MCM4Venus nuclear intensity right before the second and third divisions, again with no notable change in total cellular MCM4-Venus levels (Figure 8A and 8C). As with the first division, MCM4-Venus nuclear signal returned immediately on cytokinesis at completion of the later divisions. In the $c d c 27$ background, MCM4-Venus was localized in the nucleus in G1, but delocalized as cells entered the $c d c 27$ arrest, unlike in wild type, where MCM4-Venus delocalization lasted for only $\sim 3$ min coincident with cell division. MCM4-Venus remained diffuse over many hours (Movie2) in the $c d c 27$ background. Overall, these results indicate a metaphase dispersal of MCM4, just as was observed with MCM6 above, and suggest that the entire MCM complex may be transiently delocalized from the nucleus to the cytoplasm at metaphase.

To determine if this transient protein delocalization in metaphase is specific to the MCM proteins, we used a strain expressing a bleomycin-resistance gene fused to GFP (BleGFP). Unlike free GFP, Ble-GFP is nuclear-localized [48], presumably due to nuclear targeting of Ble. Much as with MCM4-Venus, Ble-GFP nuclear signal was sharply reduced for about $3 \mathrm{~min}$ right at the time of cell division, with no decrease in total cellular Ble-GFP signal (Movie 3). Ble-GFP in the $c d c 27$ background diffused from the nucleus as cells entered a $c d c 27$ arrest and failed to re-concentrate for the duration of the movie (Movie4). Thus, Ble-GFP reconstitutes the cell-cycle-regulated nuclear-cytoplasmic traffic observed with MCM4 and MCM6.

We were unable to analyze ORC1-Venus and CDC6-Venus by time-lapse microscopy, presumably due to their low abundance as noted above from Western analysis in Figure 6B. However, cytoplasmic diffusion of CDC6-mCherry was observed by indirect 
immunofluorescence in the $c d c 27$ mutant, instead of nuclear concentration observed in wild type (Figure 9B; see below).

\section{CDC6 protein levels are dependent on CDKA1 activity}

By immunofluorescence, CDC6-mCherry was detectable in cell nuclei, although almost never in cells with more than two nuclei. This did not reflect completion of cell divisions, since these cells, in general, underwent 3-4 division cycles (yielding 8-16 nuclei) (Figure 9A). The model from budding yeast (see Introduction) suggests that CDC6 should be required at the end of each mitosis to license replication for the succeeding rounds. We don't know if this is a real difference between the systems or if late rounds of replication in Chlamydomonas can be licensed with very low (undetectable) levels of Cdc6.

In budding yeast, Cdc6 protein is regulated by transcription, localization and protein degradation (see Introduction). Cdc6 is phosphorylated by $\mathrm{Cdk} 1$ and targeted for ubiquitination followed by proteasome-mediated protein degradation [14-16, 49-51]. Therefore, we tested if Chlamydomonas CDC6-mCherry protein levels are affected by inactivation of $C D K A 1$ or $C D K B 1$. CDC6-mCherry was transiently localized to the nucleus during the division cycle in wild type cells, and also nuclear-localized in $r n r l, c d k b l$, cycbl, orcl and mcm6-GHI mutants (Figures 9A and 9B). By contrast, in the cdkal mutant, CDC6-mCherry was completely undetectable (Figure 9B). Thus CDKA1 is required for efficient CDC6 accumulation. Unlike in yeast, where CDK activity results in destabilization and lower CDC6 levels, in Chlamydomonas the reverse is observed.

To confirm and extend these findings, we examined CDC6-mCherry protein level by western blot in various mutant backgrounds. Results were consistent with the immunofluorescence results: CDC6-mCherry was observed in $r n r 1, c d k b 1, c y c b$, orcl, mcm6-981A, mcm6-GHI and cdc27 after $12 \mathrm{hrs}$ from release (Figure 10A), but was undetectable in the cdkal mutant at 12 and $14 \mathrm{hrs}$ after release (Figure 10A). This finding was reproduced with three independent CDC6-mCherry cdkal clones, where in all cases the CDC6-mCherry transgene efficiently rescued ts-lethality of the $c d c 6-1$ mutation (data not shown). 
CDC6 is still essential in a $c d k a l$ background, since $c d k a 1$; $c d c 6-1$ double mutants are temperature-sensitive but $c d k a 1$;CDC6 single mutants are not (data not shown). This finding implies that there must be an undetectable but still functional level of CDC6 expressed in the $c d k a l$ mutant. The prolonged delay in replication in $c d k a 1$ mutants [5] may be due at least in part to very low levels of this essential replication protein.

\section{Chlamydomonas CDC6 is degraded by the proteasome}

In yeast, CDK-phosphorylated Cdc6 is degraded by ubiquitin-mediated proteolysis [14-16, 49, 51]. We examined Chlamydomonas CDC6 accumulation in the presence of a proteasome inhibitor, MG132. Cells were released from G1 and collected after 12, 14 or 16 hrs. In wild type cells, CDC6 levels were increased by MG132 treatment (Figure 10B), suggesting that CDC6 is degraded by the proteasome. However, MG132 treatment did not result in detectable CDC6 accumulation in the cdkal background (Figure 10B). Therefore, CDC6 suppression in the $c d k a l$ mutant is unlikely to be due solely to excessive proteasomal degradation. In animal cells, MG132 blocks cells in metaphase. However, in our experiments, the Chlamydomonas cell cycle profile was not significantly changed by treatment (data not shown). We do not know if failure of MG132 to arrest the Chlamydomonas cell cycle is due to inefficient proteasome inhibition at the levels we used or for some other reason. However, the cell cycle progression in MG132-treated cells does allow us to conclude that the CDC6 stabilization we observe is not due to an indirect effect of cell cycle arrest.

\section{CDC45 is nuclear and may interact with mitotic spindles}

CDC45 is a key protein required for conversion of a replication origin loaded with Mcm2-7 to a firing replication fork [12]. Cdc45-mCherry localization was analyzed by indirect immunofluorescence. We found that CDC45-mCherry was localized to the nucleus (Figure 11A). However, there was no transient CDC45 diffusion observed in any cells. In the $c d c 27$ mutant, CDC45-mCherry stayed in the nucleus and was co-localized with tubulin (mitotic spindle) staining (Figure 11B). We conclude that CDC45 was not diffused to the 
cytoplasm at metaphase, unlike MCM4, MCM6, CDC6, and ble-GFP (see above). This may be due to tethering of CDC45 to the mitotic spindle. The function of CDC45 interaction with the mitotic spindle is unknown.

\section{DISCUSSION}

\section{A conserved set of replication control proteins in the plant kingdom}

Our previous hunt for high-throughput temperature-sensitive mutants generated hundreds of cell cycle mutants, for which the causative mutations were determined by multiplexed bulked-segregant sequence analysis $[35,36]$. A number of these mutations fell in proteins aligning with replication control proteins defined in other eukaryotes. We show here that these mutants were indeed defective in DNA replication, indicating that their core functions as well as their sequences are conserved (Figure 1).

Inhibition of replication by any of these mutants also blocked mitotic spindle formation (Figure 1C). This could reflect a checkpoint preventing mitotic entry until completion of replication as observed in many other eukaryotes [38]. A checkpoint of this kind may thus be conserved in Chlamydomonas, and probably in the plant kingdom overall [52]. In contrast, another well-known checkpoint mechanism, the spindle assembly checkpoint which blocks mitotic exit and the next round of DNA replication upon spindle disruption, is weak or entirely absent in Chlamydomonas [35].

\section{Complementation of temperature-sensitive lethal mutations with fluorescent}

\section{transgenes.}

We generated universal plasmids containing fluorescent tags, into which we inserted wild-type sequences to rescue the corresponding ts mutants. Using the rescued strains, we analyzed protein expression and localization (Figure 7,9 and 11). We used $m$ Cherry tagged strains for indirect immunofluorescence because anti-mCherry antibody generally works better than anti-GFP antibody. For time-lapse microscopy, mCherry signal is hard to detect due to chloroplast autofluorescence in Chlamydomonas, but cell-cycleregulated accumulation and localization of MCM4-Venus was clearly detectable at high 
time resolution (Figure 8).

\section{A loss of nuclear retention in a "closed mitosis"}

Whether the nuclear envelope breaks down in mitosis or remains intact ('open' vs. 'closed' mitosis) is a significant phylogenetic marker, with multiple independent transitions in different lineages [53]. Chlamydomonas is traditionally classified as having a closed mitosis; however, permeability of the nuclear envelope in a closed mitosis may vary among organisms [54]. In Chlamydomonas, MCM4 and MCM6 were localized to the nucleus throughout the division cycle except during mitosis, when they transiently diffused to the cytoplasm; CDC6 may show a similar localization pattern (Figure 7A, 8A, 9C). Transient MCM protein diffusion during mitosis is also observed in Arabidopsis [31], which has an open mitosis.

Chlamydomonas spindle poles are outside the nuclear envelope and the spindle enters the nucleus through large holes called polar fenestrae [55]. The fenestrae are 300500um in diameter, 5 times larger than nuclear pores in interphase. If the fenestrae are truly open channels, this should allow rapid diffusion of all nuclear components that are not tightly bound to large structures (such as chromosomes, mitotic spindles or a nuclear matrix). This transient protein diffusion might therefore be functionally equivalent to nuclear membrane breakdown in organisms showing 'open mitosis'. Nuclear envelope breakdown is triggered in early mitosis and nuclei are reassembled at the end of mitosis. In an early proposal to explain 'licensing' of replication origins in Xenopus, it was proposed that the nuclear envelope excludes access of key replication factors to fired origins; nuclear envelope breakdown allows access and subsequent origin licensing [56]. We speculate that the fenestrae in Chamydomonas may serve as a related cell cycle reset mechanism. Our results suggest that the classic distinction between 'open' and 'closed' mitosis may not imply restricted traffic of nuclear components [54]. A general loss of nuclear integrity, rather than a specific effect on replication proteins, is suggested by transient diffusion of ble-GFP from the nucleus during mitosis (see above).

In contrast, CDC45-mCherry was stably nuclear throughout the cell cycle. In 
metaphase, when other proteins diffused through the cytoplasm, CDC45 appeared colocalized to spindles (Figure 11B); this anchoring may prevent CDC45 cytoplasmic diffusion in metaphase. We do not yet know the functional significance of CDC45 spindle localization.

\section{Pre-RC formation in Chlamydomonas}

All of the DNA replication proteins we analyzed were essentially absent during the long G1 before multiple fission (Figure 6). This is correlated with transcriptional profiles of corresponding genes $[5,6]$. In yeast, $\mathrm{CDK}$ activity is very low specifically in late mitosis and early G1, and low CDK activity is permissive for pre-RC formation and origin loading with the MCM helicase; loaded origins persist through G1 until firing occurs under positive CDK control. The essential absence of pre-RC proteins in Chlamydomonas during G1 suggests that origin loading may be regulated differently than in yeast, since during most of G1 there appears to be negligible levels of pre-RC proteins; however, we lack a direct origin loading assay to pursue this at present. In yeast, such studies were greatly facilitated by specific origin sequences (ARS consensus). We do not know if Chlamydomonas has specific origin sequences, or, by contrast, if origin selection is largely sequence-nonspecific as in animal cells.

In yeast, $\mathrm{CDK}$ phosphorylates the MCM complex, which triggers its nuclear export during S-phase after individual origins have fired. Our results show that MCM6 stays in the nucleus in $c d k a l$ and $c d k b 1$ mutants (Figure 7), which could be consistent with this mechanism. However, in wild-type cells MCM4 stays in the nucleus throughout the cell cycle except for a window of $\sim 3$ min, indicating that CDK probably does not exclude MCM4 from the nucleus during S-phase in Chlamydomonas, in contrast to results in yeast.

CDC6 also stayed in the nucleus in the $c d k b 1$ mutant (Figure 9). However, CDC6 was undetectable in the $c d k a l$ mutant (Figure 9 and 10). This was a surprising result because Cdc6 degradation is mediated through CDK-dependent phosphorylation in yeast. $C D K A 1$ expression peaked during S-phase 1 hour before $C D K B 1$ comes up [5, 6]. Efficient and timely expression of DNA replication genes depends on CDKA1 [5]. The Cdc6 
suppression in $c d k a l$ mutant might be a reflection of this transcriptional inhibition. Cdc6 regulation is diverse across the species. In budding yeast, CDK mediates SCF-dependent Cdc6 degradation to inhibit pre-RC formation in S-phase. In humans, APC-dependent Cdc6 proteolysis inhibits Cdc6 accumulation, and CDK phosphorylates and protects $\mathrm{Cdc} 6$ to trigger origin licensing [23]. It is unclear whether any of these mechanisms will apply in Chlamydomonas. Proteasome-dependent degradation of CDC6 may provide a first clue (Figure 10B).

Chlamydomonas ts-lethal mutations in many proteins controlling DNA replication allow a detailed functional examination of DNA replication control in the plant kingdom. Absence of an accessible culture system or conditional alleles, and a high level of gene duplication makes such experimentation (and even identification of the cognate proteins; see above) much more difficult in land plants. As expected from phylogenetic relationships, conserved Chlamydomonas proteins are more closely related to land plant proteins than to the orthologous yeast or animal proteins (Figure 2). This closer conservation likely extends to functional considerations as well; thus Chlamydomonas may be a valuable model for replication control in the critically important plant kingdom.

The budding yeast DNA replication control system has been characterized in depth and detail, providing specific molecular hypotheses to evaluate in Chlamydomonas. The results reported here already demonstrate conserved features but also some likely and significant differences. It is important to note that conservation of sequence alone does not guarantee conservation of function or regulation. Conditional mutants are key resources to rapidly deliver functional analysis.

Sequence comparisons indicate that in some cases, Chlamydomonas and human replication proteins are much more homologous than the corresponding yeast and human proteins are (for example, DIV20/RecQL4/SLD2 discussed above). This is a genomic feature applying to many conserved proteins and presumably reflects rapid sequence evolution in the yeast lineages [57]. Whereas Chlamydomonas molecular evolution is quite slow, leading to retention of sequences and features found in animals but not in yeast [58]. Thus, although clearly in its infancy compared to the highly developed yeast systems, study 
of Chlamydomonas replication may provide useful models for features found in animals but lost in yeast.

Table 1

\begin{tabular}{|c|c|c|c|c|c|}
\hline & & $\begin{array}{l}\text { Best } \\
\text { BLAST } \\
\text { score } \\
\text { against: }\end{array}$ & & & NOTES \\
\hline Gene & C.r. transcript & A.th. & M.m. & S.c. & \\
\hline ORC1 & Cre10.g455600.t1.1 & 337 & 202 & 137 & \\
\hline CDC6 & Cre06.g292850.t1.2 & 139 & 127 & 112 & \\
\hline MCM4 & Cre07.g316850.t1.2 & 681 & 560 & 582 & $\begin{array}{l}\text { A.t. listed as MCM2/5/7 family } \\
\text { protein }\end{array}$ \\
\hline MCM6 & Cre03.g178650.t1.1 & 734 & 619 & 595 & $\begin{array}{l}\text { A.t. listed as MCM2/5/7 family } \\
\text { protein }\end{array}$ \\
\hline MCM7 & Cre10.g455850.t1.2 & 704 & 594 & 609 & $\begin{array}{l}\text { A.t. listed as } M C M 2 / 5 / 7 \text { family } \\
\text { protein }\end{array}$ \\
\hline CDC45 & Cre06.g270250.t1.1 & 235 & 159 & $\mathrm{NOHIT}$ & \\
\hline topBP1 & Cre12.g515900.t1.2 & 216 & 117 & NO HIT & $\begin{array}{l}\text { A.t. listed as 'transcriptional } \\
\text { activator'; no hit against S.c. } \\
\text { DPB11 }\end{array}$ \\
\hline RecQL4 & Cre15.g634701.t1.1 & 258 & 162 & NO HIT & $\begin{array}{l}\text { A.t. listed as DEAD/DEAH RNA } \\
\text { helicase protein; no hit against S.c } \\
\text { SLD2 }\end{array}$ \\
\hline RNR1 & Cre12.g492950.t1.2 & 1278 & 1160 & 1104 & \\
\hline CDC27 & Cre17.g740510.t1.1 & 397 & 242 & 156 & \\
\hline
\end{tabular}

\section{MATERIALS AND METHODS}

\section{Cell culture conditions}

All strains are listed in Table S1. Cells were maintained on Tris-acetate-phosphate (TAP) medium (Harris, 2008) at $21^{\circ} \mathrm{C}$ under continuous illumination at $~ 100$ PAR (determined with Apogee Quantum Light Meter). In order to synchronize cells in G1 by nitrogen depletion, cells were spread on $0.1 \times N$ TAP plates (containing 1/10 of standard TAP levels of ammonium chloride) and incubated for 2 days at $21^{\circ} \mathrm{C}$ under the light. The G1 arrest was released by plating cells on TAP plates at $33^{\circ} \mathrm{C}$ under continuous illumination at $\sim 150$ PAR.

\section{Strain cross and mutant analysis}

Strain cross was performed as described previously [59]. Strain list is in Table S1. 
Tetrad haploid progenies were dissected with a Zeiss Axioskop 40 Tetrad microscope. Mutations were verified by allele-specific competitive PCR (Onishi M). Briefly, three primers were designed for each mutant. Two forward primers were designed to distinguish wild type and mutant by a length of PCR product. Forward primer 1 contains wild type sequence with $20 \mathrm{bp}$ long. Forward primer 2 contains the mutant sequence with $40 \mathrm{bp}$ long. A reverse primer was designed to recognize sequence a few hundred base pairs downstream of the mutant site. PCR reaction was performed using these 3 primers, and the PCR product was analyzed using 3\% SB agarose gel. The PCR band with the mutation is $20 \mathrm{bp}$ longer than that with wild type. Primer sequences are listed in Table S2.

\section{Plasmid construction and transformation}

The mCherry plasmid FC1011 plasmid was constructed by NEBuilder HiFi DNA assembly Master Mix (New England Biolabs, MA) using pKA1 (CDKB plasmid) as a temperate [41]. The mCherry fragment was replaced by GFP and Venus to create GFP- and Venus-universal plasmids, named FC1012 and FC1001 using multiple cloning sites. The desired gene (MCM6, CDC45, or CDC6) was amplified from genomic DNA and inserted into the mCherry plasmid at the multiple cloning site. ORC1-mCherry and MCM4-VENUS plasmids were synthesized by SynBio (NJ). For these plasmids, all of the introns except the first two and the last intron were removed. The plasmid linearized by XbaI digestion was used for transformation.

Temperature sensitive mutant cells were transformed with the linearized plasmid by electroporation ( $500 \mathrm{~V}, 50 \mu \mathrm{F}, 800 \square \Omega$ ) using GenePulser Xcell (Bio-Rad, CA). The cells were recovered in TAP containing $40 \square \mathrm{mM}$ sucrose, incubated under light overnight, and then plated on a TAP plate containing $10 \mathrm{ug} / \mathrm{ml}$ paromomycin. Complementation of temperature sensitivity among paromomycin-resistant transformants was determined by serial dilution of cells on TAP plate at $33^{\circ} \mathrm{C}$ for 2-5 days. Plasmid integration and structure was confirmed by PCR.

\section{Flow Cytometry}


Flow Cytometry analysis (FACS) was performed as described by BD Accuri C6 instrument (BD Biosciences)[35]. The data was analyzed by FlowJo software (FlowJo, LLC, OR).

\section{Western Blotting}

Cells were lysed in RIPA buffer containing protease inhibitors with glass beads using FastPrep (MP Biomedicals, CA) for 20s, twice, at speed 6. Cleared cell lysate was denatured with SDS buffer and separated by SDS-PAGE with Novex 4-12\% Tris-glycine polyacrylamide gel (Invitrogen, Life Technologies, CA). Western blot analysis was performed using anti-mCherry antibody (16D7) at 1:2000 dilution (Thermo Fisher, MA), anti-GFP antibody (GF28R) at 1:2000 dilution (Thermo Fisher, MA) and anti-AtpB antibody at 1:5000 dilution (Agrisera, Sweden) as a loading control. HRP-conjugated secondary antibody was used to detect signal with SuperSignal West Femto (Thermo Fisher, MA). Image was acquired with Image Quant LAS 4000 (GE Healthcare, IL). The band intensity was quantified with ImageJ (NIH).

\section{Indirect Immunofluorescence}

Indirect immunofluorescence was performed as described [35]. The primary antibodies are anti-mCherry monoclonal antibody (16D7) at 1:200 dilution (Thermo Fisher, MA) and anti- $\alpha$-tubulin antibodies (clone B-5-1-2, Sigma-Aldrich) at 1:5000. The secondary antibody was anti-rat-Alexa 568 (Thermo Fisher, MA) at 1:1000 and DyLight ${ }^{\mathrm{TM}}$ 405 goat anti-mouse antibody (BioLegend, CA) at 1:2000, respectively. Cells were stained with $1 \mu \mathrm{M}$ or $0.5 \mu \mathrm{M}$ Sytox Green (Invitrogen, CA). Images were collected using an inverted Zeiss LSM 510 Laser Scanning confocal microscope. Images in Figure S1 were collected using DeltaVision microscope. Images were exported in TIFF format, merged and analyzed using ImageJ (NIH).

\section{Time-Lapse Microscopy}


Cells arrested in G1 by nitrogen deprivation were transferred to agarose pads and imaged with a 63X objective on a Leica DMI6000B microscope with the objective and stage heated to $33^{\circ} \mathrm{C}$. Pad architecture and other procedures were based on the budding yeast setup of Di Talia et al, but with modifications for Chlamydomonas to be described elsewhere (KP and FC, unpublished data)[46]. Images were acquired using custom software, as previously described for yeast timelapse [47], modified to adjust autofocus for Chlamydomonas (KP and FC, unpublished). Deconvolution was employed to subtract the chloroplast-specific signal in the Venus channel (KP and FC, unpublished). All images in a given movie were analyzed identically.

\section{SUPPLEMENTAL DATA}

The following materials are available in the online version of this article.

Supplemental Table 1. C. reinhardtii strains used in this paper.

Supplemental Table 2. Oligonucleotides sequence used to verify the mutation. Lowercase letters indicate additional external sequence.

\section{ACKNOWLEDGMENTS}

We thank Daria Postavnaia for help with making plasmids in Figure 4A. We also thank Alison North at The Rockefeller University Bio-Imaging Resource Center for technical assistance with confocal microscopy, and The Rockefeller University Flow Cytometry Resource Center for FACS analysis. This work was supported by NIH 5SC1GM121242 to AI and 2R01GM078153 to FC.

1. Morgan, D. 0. (1995). Principles of CDK regulation. Nature 374, 131-134.

2. Cross, F. R., and Umen, J. G. (2015). The Chlamydomonas cell cycle. Plant J 82, 370-392. 
3. Vandepoele, K., Raes, J., De Veylder, L., Rouze, P., Rombauts, S., and Inze, D. (2002). Genome-wide analysis of core cell cycle genes in Arabidopsis. Plant Cell 14, 903-916.

4. Cizkova, M., Pichova, A., Vitova, M., Hlavova, M., Hendrychova, J., Umysova, D., Galova, E., Sevcovicova, A., Zachleder, V., Umen, J. G., et a1. (2008). CDKA and CDKB kinases from Chlamydomonas reinhardtii are able to complement cdc28

temperature-sensitive mutants of Saccharomyces cerevisiae. Protoplasma 232, 183191.

5. Tulin, F., and Cross, F. R. (2015). Cyclin-Dependent Kinase Regulation of Diurnal Transcription in Chlamydomonas. Plant Cel1 27, 2727-2742.

6. Zones, J. M., Blaby, I. K., Merchant, S. S., and Umen, J. G. (2015). High-Resolution Profiling of a Synchronized Diurnal Transcriptome from Chlamydomonas reinhardtii Reveals Continuous Cell and Metabolic Differentiation. Plant Ce11 27, 2743-2769.

7. Be11, S. P., and Stillman, B. (1992). ATP-dependent recognition of eukaryotic origins of DNA replication by a multiprotein complex. Nature 357, 128-134.

8. Liang, C., Weinreich, M., and Stillman, B. (1995). ORC and Cdc6p interact and determine the frequency of initiation of DNA replication in the genome. Cel1 81, $667-676$.

9. Cocker, J.H., Piatti, S., Santocanale, C., Nasmyth, K., and Diffley, J. F. (1996). An essential role for the Cdc6 protein in forming the pre-replicative complexes of budding yeast. Nature 379, 180-182.

10. Chen, S., de Vries, M. A., and Be11, S.P. (2007). Orc6 is required for dynamic recruitment of Cdt1 during repeated Mcm2-7 loading. Genes Dev 21, 2897-2907.

11. Remus, D., and Diffley, J.F. (2009). Eukaryotic DNA replication control: 1ock and load, then fire. Curr Opin Ce11 Biol 21, 771-777.

12. Tanaka, S., and Araki, H. (2013). Helicase activation and establishment of replication forks at chromosomal origins of replication. Cold Spring Harb Perspect Biol 5, a010371.

13. Diffley, J.F. (1996). Once and only once upon a time: specifying and regulating origins of DNA replication in eukaryotic cells. Genes Dev 10, 2819-2830.

14. Drury, L. S., Perkins, G., and Diffley, J.F. (2000). The cyclin-dependent kinase Cdc28p regulates distinct modes of Cdc6p proteolysis during the budding yeast cell cycle. Curr Biol 10, 231-240.

15. Drury, L.S., Perkins, G., and Diffley, J.F. (1997). The Cdc4/34/53 pathway targets Cdc6p for proteolysis in budding yeast. EMBO J 16, 5966-5976.

16. Perkins, G., Drury, L. S., and Diffley, J.F. (2001). Separate SCF (CDC4) recognition elements target $\mathrm{Cdc6}$ for proteolysis in $\mathrm{S}$ phase and mitosis. EMB0 J $20, \quad 4836-4845$.

17. E1sasser, S., Lou, F., Wang, B., Campbe11, J. L., and Jong, A. (1996). Interaction between yeast Cdc6 protein and B-type cyclin/Cdc28 kinases. Mol Biol Cell 7, $1723-1735$.

18. DePamphilis, M. L. (2005). Cell cycle dependent regulation of the origin recognition complex. Cell Cycle 4, 70-79.

19. Fujita, M., Yamada, C., Tsurumi, T., Hanaoka, F., Matsuzawa, K., and Inagaki, M. (1998). Cell cycle- and chromatin binding state-dependent phosphorylation of human MCM heterohexameric complexes. A role for cdc2 kinase. J Biol Chem 273, 
$17095-17101$.

20. Moritani, M. and Ishimi, Y. (2013). Inhibition of DNA binding of MCM2-7 complex by phosphorylation with cyclin-dependent kinases. J Biochem 154, 363-372.

21. Fujita, M., Yamada, C., Goto, H., Yokoyama, N., Kuzushima, K., Inagaki, M., and Tsurumi, T. (1999). Ce11 cycle regulation of human CDC6 protein. Intracellular localization, interaction with the human mcm complex, and CDC2 kinase-mediated hyperphosphorylation. J Biol Chem 274, 25927-25932.

22. Walter, D., Hoffmann, S., Komseli, E. S., Rappsilber, J., Gorgoulis, V., and Sorensen, C. S. (2016). SCF (Cyclin F)-dependent degradation of CDC6 suppresses DNA re-replication. Nat Commun 7, 10530.

23. Mailand, N., and Diffley, J.F. (2005). CDKs promote DNA replication origin licensing in human cells by protecting $\mathrm{Cdc} 6$ from $\mathrm{APC} / \mathrm{C}-$ dependent proteolysis. Ce11 122, 915-926.

24. Duursma, A.M., and Agami, R. (2005). CDK-dependent stabilization of Cde6: 1inking growth and stress signals to activation of DNA replication. Cell Cycle 4, 17251728.

25. Tanaka, S., Umemori, T., Hirai, K., Muramatsu, S., Kamimura, Y., and Araki, H. (2007). CDK-dependent phosphorylation of S1d2 and S1d3 initiates DNA replication in budding yeast. Nature 445, 328-332.

26. Zegerman, P., and Diffley, J.F. (2007). Phosphorylation of S1d2 and S1d3 by cyclin-dependent kinases promotes DNA replication in budding yeast. Nature 445, 281-285.

27. Garcia, V., Furuya, K., and Carr, A. M. (2005). Identification and functional analysis of TopBP1 and its homologs. DNA Repair (Amst) 4, 1227-1239.

28. Masuda, H. P., Ramos, G. B., de Almeida-Engler, J., Cabral, L. M. , Coqueiro, V. M. , Macrini, C.M., Ferreira, P.C., and Hemerly, A.S. (2004). Genome based identification and analysis of the pre-replicative complex of Arabidopsis thaliana. FEBS Lett 574, 192-202.

29. de Jager, S. M., Menges, M., Bauer, U. M. , and Murra, J.A. (2001). Arabidopsis E2F1 binds a sequence present in the promoter of S-phase-regulated gene AtCDC6 and is a member of a multigene family with differential activities. Plant Mol Biol 47, $555-568$.

30. Castellano, M. M., del Pozo, J.C., Ramirez-Parra, E., Brown, S., and Gutierrez, C. (2001). Expression and stability of Arabidopsis CDC6 are associated with endoreplication. Plant Ce11 13, 2671-2686.

31. Shultz, R.W., Lee, T. J., Allen, G. C., Thompson, W. F., and Hanley-Bowdoin, L. (2009). Dynamic localization of the DNA replication proteins MCM5 and MCM7 in plants. Plant Physio1 150, 658-669.

32. Springer, P. S., Holding, D. R., Groover, A., Yordan, C., and Martienssen, R. A. (2000). The essential Mcm7 protein PROLIFERA is localized to the nucleus of dividing cells during the $G(1)$ phase and is required maternally for early Arabidopsis development. Development 127, 1815-1822.

33. Dresselhaus, T., Srilunchang, K. 0., Le1jak-Levanic, D., Schreiber, D. N., and Garg, P. (2006). The fertilization-induced DNA replication factor MCM6 of maize shuttles between cytoplasm and nucleus, and is essential for plant growth and development. Plant Physiol 140, 512-527. 
34. Tuteja, N., Tran, N. Q., Dang, H. Q., and Tuteja, R. (2011). Plant MCM proteins: role in DNA replication and beyond. Plant Mol Biol 77, 537-545.

35. Tulin, F., and Cross, F. R. (2014). A microbial avenue to cell cycle control in the plant superkingdom. Plant Ce11 26, 4019-4038.

36. Breker, M., Lieberman, K., and Cross, F. R. (2018). Comprehensive Discovery of Ce11-Cycle-Essential Pathways in Chlamydomonas reinhardtii. Plant Ce11 30, 11781198.

37. Kamimura, Y., Tanaka, H., Kobayashi, Y., Shikanai, T., and Nishimura, Y. (2018). Chloroplast nucleoids as a transformable network revealed by live imaging with a microfluidic device. Commun Biol 1, 47.

38. Hartwe11, L. H., and Weinert, T.A. (1989). Checkpoints: controls that ensure the order of cell cycle events. Science 246, 629-634.

39. Duncker, B.P., Chesnokov, I.N., and McConkey, B. J. (2009). The origin recognition complex protein family. Genome Biol 10, 214.

40. Sangrithi, M. N., Bernal, J. A., Madine, M., Philpott, A., Lee, J., Dunphy, W. G., and Venkitaraman, A. R. (2005). Initiation of DNA replication requires the RECQL4 protein mutated in Rothmund-Thomson syndrome. Ce11 121, 887-898.

41. Atkins, K. C. , and Cross, F. R. (2018). Interregulation of CDKA/CDK1 and the PlantSpecific Cyclin-Dependent Kinase CDKB in Control of the Chlamydomonas Cell Cycle. Plant Ce11 30, 429-446.

42. King, R. W., Peters, J.M., Tugendreich, S., Rolfe, M., Hieter, P., and Kirschner, M. W. (1995). A 20 S complex containing CDC27 and CDC16 catalyzes the mitosisspecific conjugation of ubiquitin to cyclin B. Cell 81, 279-288.

43. Nguyen, V. Q., Co, C., and Li, J. J. (2001). Cyclin-dependent kinases prevent DNA re-replication through multiple mechanisms. Nature 411, 1068-1073.

44. Liku, M. E., Nguyen, V. Q., Rosales, A. W., Irie, K., and Li, J. J. (2005). CDK phosphorylation of a nove1 NLS-NES module distributed between two subunits of the Mcm2-7 complex prevents chromosomal rereplication. Mo1 Biol Ce11 16, 5026-5039.

45. Nguyen, V. Q., Co, C., Irie, K., and Li, J. J. (2000). C1b/Cdc28 kinases promote nuclear export of the replication initiator proteins Mcm2-7. Curr Biol 10, 195205.

46. Di Talia, S., Skotheim, J.M., Bean, J.M., Siggia, E. D., and Cross, F. R. (2007). The effects of molecular noise and size control on variability in the budding yeast cell cycle. Nature 448, 947-951.

47. Charvin, G., Cross, F. R., and Siggia, E. D. (2008). A microfluidic device for temporally controlled gene expression and long-term fluorescent imaging in unperturbed dividing yeast cel1s. PLoS One 3, e1468.

48. Fuhrmann, M. , Oerte1, W., and Hegemann, P. (1999). A synthetic gene coding for the green fluorescent protein (GFP) is a versatile reporter in Chlamydomonas reinhardtii. Plant J 19, 353-361.

49. Calzada, A., Sanchez, M., Sanchez, E., and Bueno, A. (2000). The stability of the Cdc6 protein is regulated by cyclin-dependent kinase/cyclin B complexes in Saccharomyces cerevisiae. J Bio1 Chem 275, 9734-9741.

50. Sanchez, M., Calzada, A., and Bueno, A. (1999). The Cdc6 protein is ubiquitinated in vivo for proteolysis in Saccharomyces cerevisiae. J Biol Chem 274, 9092-9097.

51. E1sasser, S., Chi, Y., Yang, P., and Campbe11, J. L. (1999). Phosphorylation 
controls timing of Cdc6p destruction: A biochemical analysis. Mol Biol Ce11 10, 3263-3277.

52. Culligan, K., Tissier, A., and Britt, A. (2004). ATR regulates a G2-phase ce11cycle checkpoint in Arabidops is thaliana. Plant Ce11 16, 1091-1104.

53. Sazer, S., Lynch, M., and Needleman, D. (2014). Deciphering the evolutionary history of open and closed mitosis. Curr Biol 24, R1099-1103.

54. De Souza, C.P., and 0smani, S.A. (2007). Mitosis, not just open or closed. Eukaryot Ce11 6, 1521-1527.

55. Johnson, U.G., and Porter, K. R. (1968). Fine structure of cell division in Chlamydomonas reinhardi. Basal bodies and microtubules. J Cell Biol 38, 403-425.

56. Blow, J. J., and Laskey, R. A. (1988). A role for the nuclear envelope in controlling DNA replication within the cell cycle. Nature 332, 546-548.

57. Burki, F., Shalchian-Tabrizi, K., and Pawlowski, J. (2008). Phylogenomics reveals a new 'megagroup' including most photosynthetic eukaryotes. Biol Lett 4, 366-369.

58. Merchant, S. S., Prochnik, S.E., Vallon, 0., Harris, E. H., Karpowicz, S. J. , Witman, G. B., Terry, A., Salamov, A., Fritz-Laylin, L.K., Marechal-Drouard, L., et a1. (2007). The Chlamydomonas genome reveals the evolution of key animal and plant functions. Science 318, 245-250.

59. Dutcher, S.K. (1995). Mating and tetrad analysis in Chlamydomonas reinhardtii. Methods Cel1 Biol 47, 531-540. 

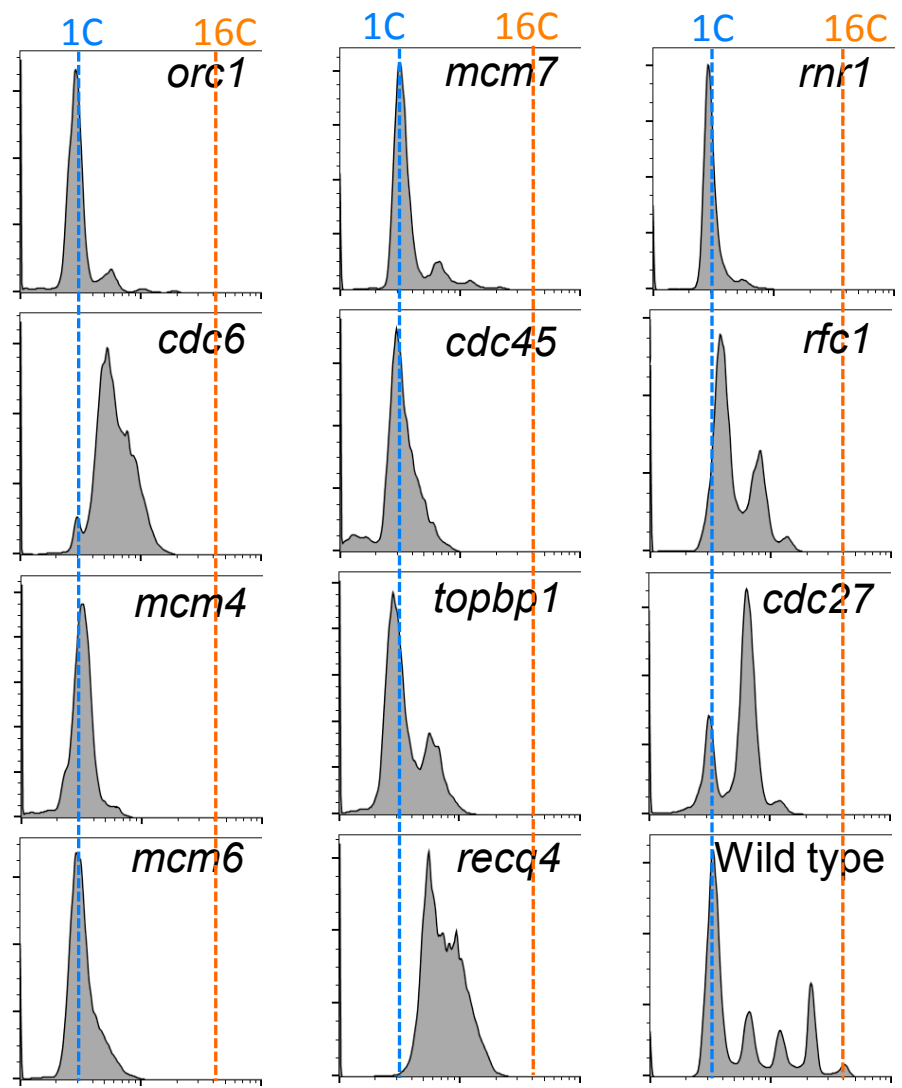

B

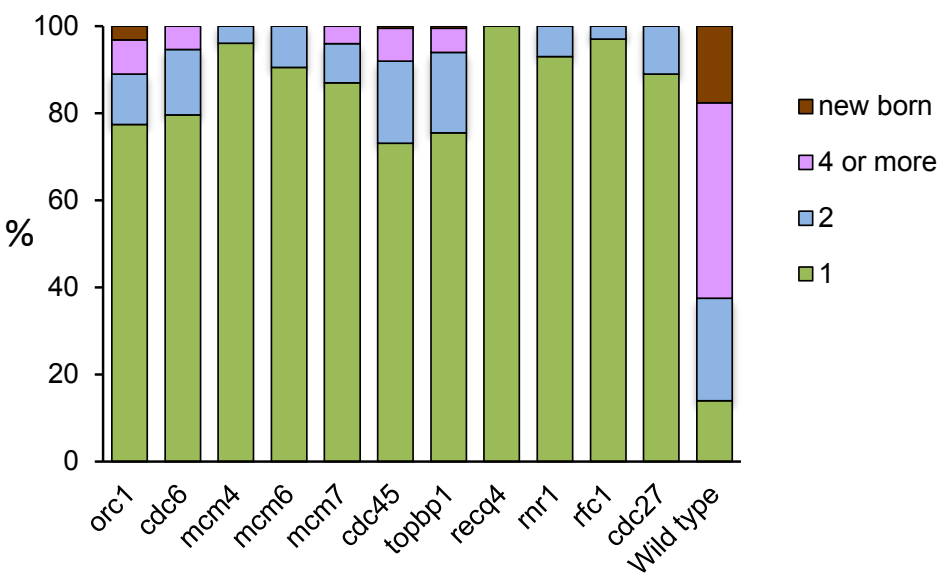

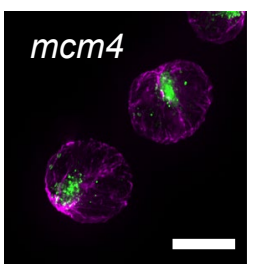
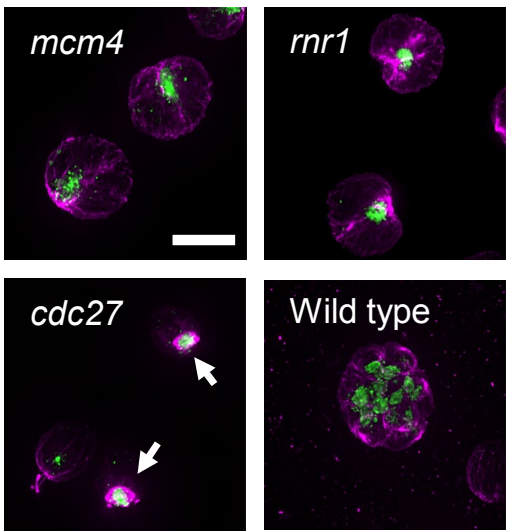

\section{Wild type}

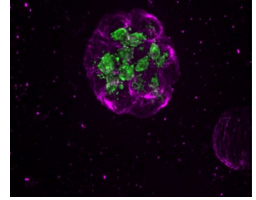

Green: Sytox Green (DNA) Magenta: Tubulin

Figure 1. Analysis of DNA replication mutants (A) Wild type cells were arrested in G1 by nitrogen starvation and the cell cycle was released for $12 \mathrm{hrs}$ at the non-permissive temperatures $33^{\circ} \mathrm{C}$. Cells were fixed and stained with Sytox for FACS. (B) Numbers of cells with new born, 4 or more nuclei, 2 nuclei or 1 nucleus was counted using the samples from A. 100 cells were counted and the percentage is shown. (C) . Inhibition of spindle formations in DNA replication mutants. Indicated temperature sensitive mutants and Wild type cells were arrested in G1 and the cell cycle was released for 12 hours at 33 degrees. Cells were fixed and stained with anti-tubulin antibody (Magenta) and Sytox Green (Green). Images were taken by DeltaVision microscope. White arrows show mitotic spindles. Bars $=10 \mu \mathrm{m}$ 

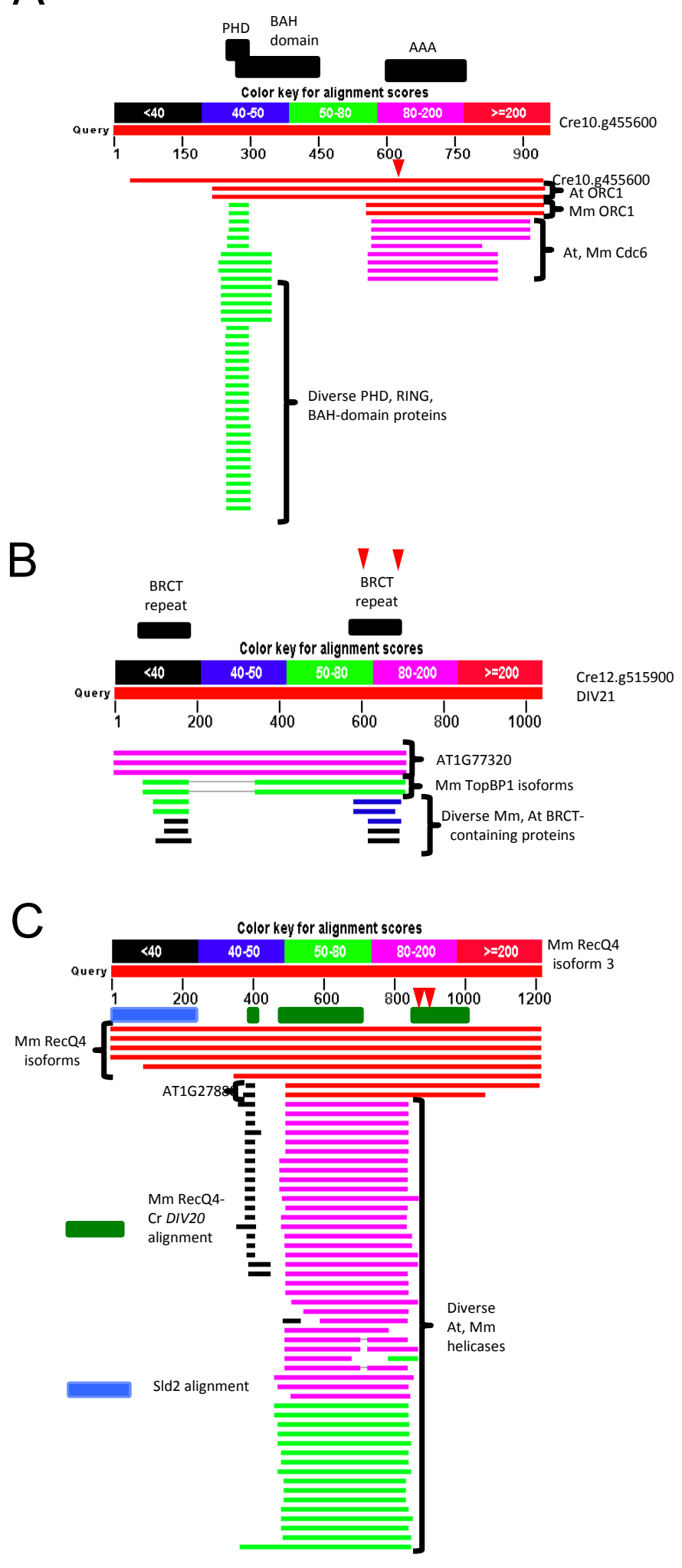

Figure 2. A graphical summary of sequence alignment by BLAST (A) Chlamydomonas Orc1 candidate, Cre10.g455600, was aligned with AtOrc1, MmOrc1, At Cdc6, MmCdc6 and proteins with PHD, RING and BAH domains. Red arrow indicates the location of div74-1 mutation. (B) Chlamydomonas TopBP1 candidate, Cre12.g515900, was aligned with AT1G77320, MmTopBP1 and proteins with Mm, At BRCT domains. Black bar on the top shows BRCT repeat. Red arrows indicate the location of div21-1 and of div21-2 mutations. (C) Mm RecQ4 candidate was aligned with MmRecQ4 isofroms, AT1G2788, and At, Mm helicases. Blue bar shows Sld2 alignment. Green bar shows an alignment with $C r$ DIV 20 gene. Red arrows indicate the location of div20-1 and of div20-2 mutations. 
$m c m 6-981 A$

mom6-GHI
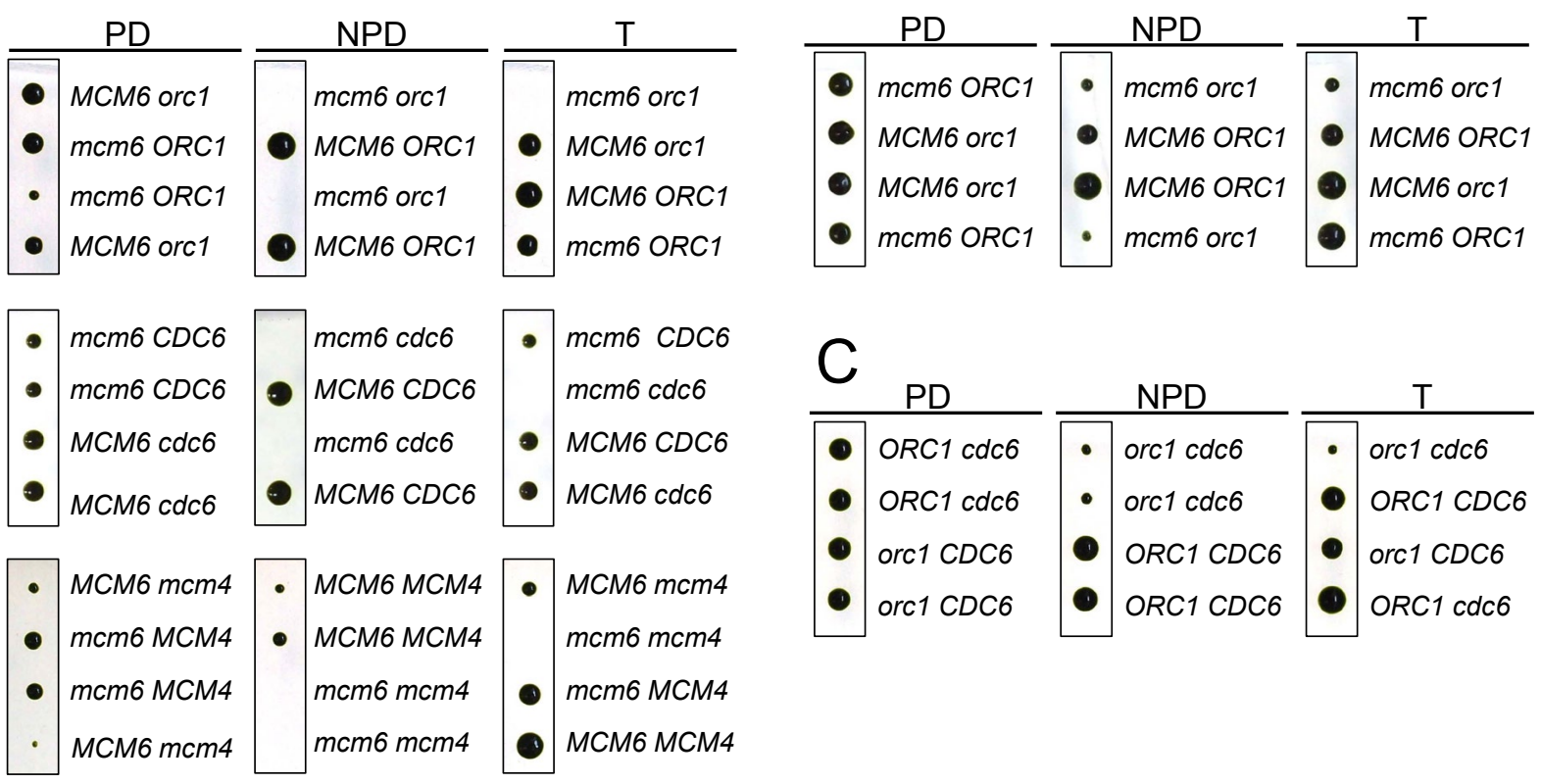

$\mathrm{D}$

\begin{tabular}{|c|c|c|c|c|c|}
\hline & orc1 & cdc6 & mcm4 & $\begin{array}{c}\text { mcm6- } \\
981 \mathrm{~A}\end{array}$ & $\begin{array}{c}\text { mom6- } \\
\text { GHI }\end{array}$ \\
\hline orc1 & & semi & No & lethal & semi \\
\hline Cdc6 & & No & lethal & No \\
\hline mcm4 & & & & lethal & ND \\
\hline $\begin{array}{c}\text { mom6- } \\
981 \mathrm{~A}\end{array}$ & & & & & ND \\
\hline $\begin{array}{c}\text { mom6- } \\
\text { GHI }\end{array}$ & & & & & \\
\hline
\end{tabular}

Figure 3. Synthetic lethality between DNA replication mutants (A) $m c m 6-981 A$ temperature sensitive mutant was crossed with orcl, $c d c 6$ or $m c m 4$ mutant. Tetrads are shown with tetra type. An example of parental ditype (PD), non-parental ditype (NPD) or tetra type (T) is shown. (B-C) Tetrad analysis of mcm6-GHI orcl and orcl cdc6 are shown. (D) A summary table of synthetic lethality tested in this study. Lethal: complete lethal, Semi: mild lethality, No: No genetic interaction, ND: No data. 


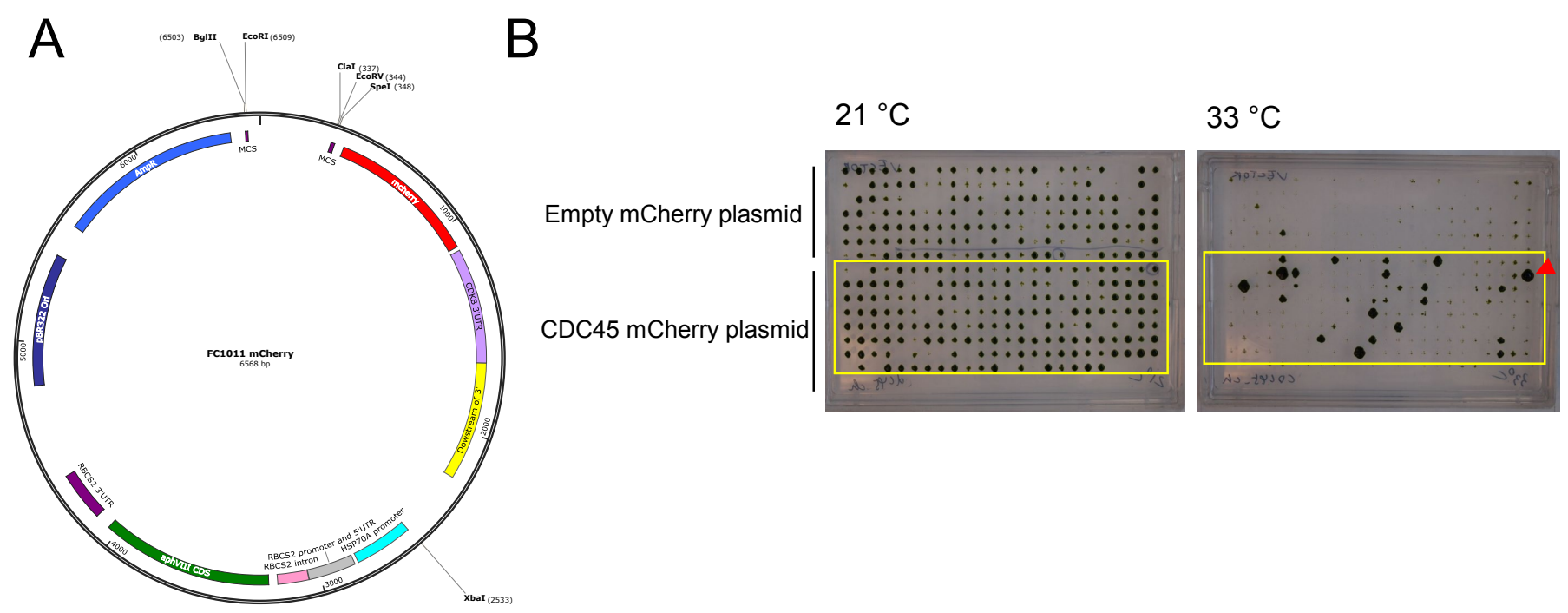

Figure 4. Chlamydomonas plasmid construction. (A) An universal mCherry plasmid map is shown. Tagging can be mCherry, Venus or GFP. (B) CDC45-mCherry plasmid was constructed and transformed into $c d c 45$ temperature sensitive mutant. Transformants were picked from plates at $21^{\circ} \mathrm{C}$ and aligned as 364 well format. The plates were incubated at 21 or $33{ }^{\circ} \mathrm{C}$ for 10 days. The colony with red arrow was further analyzed. 

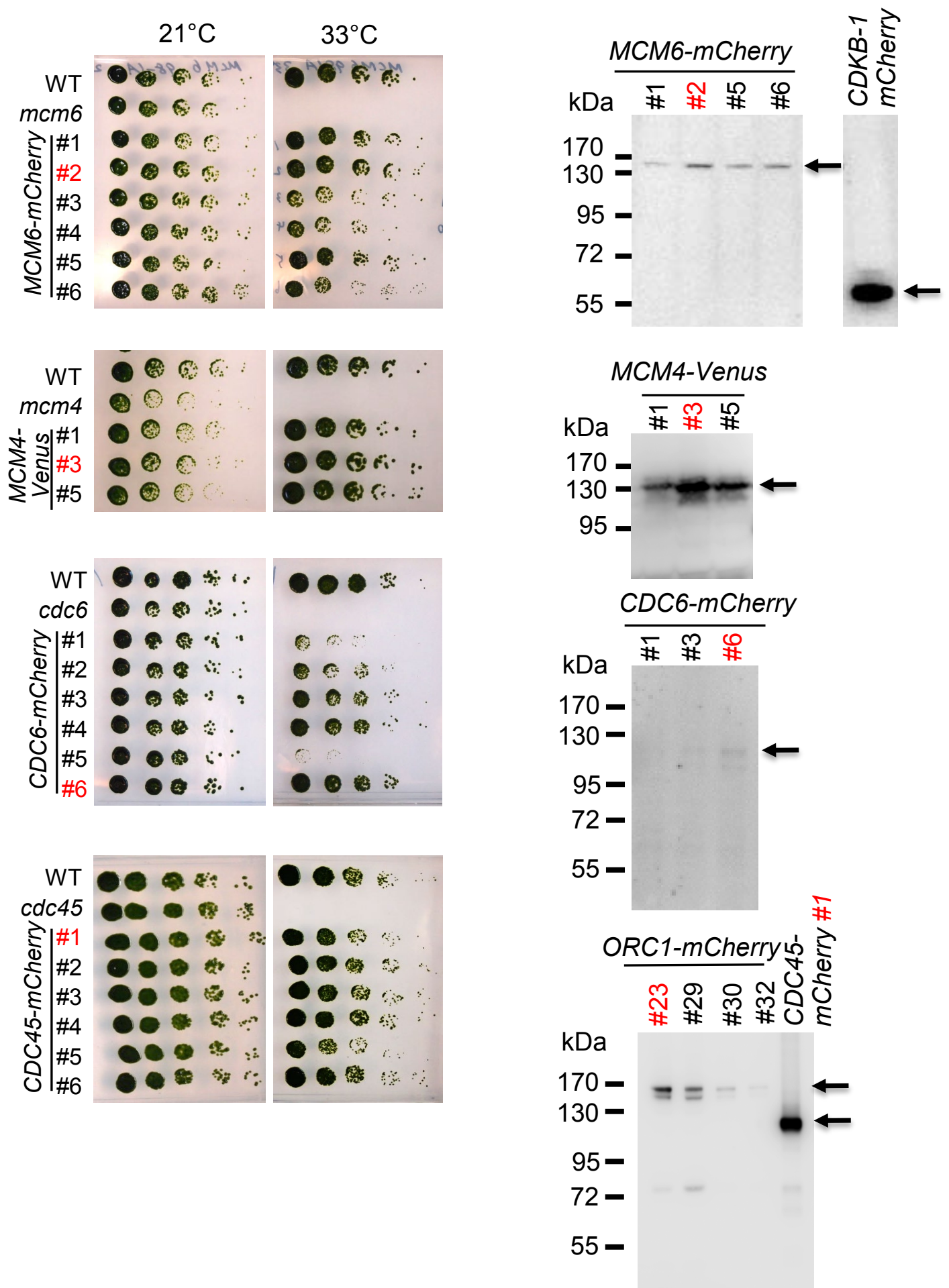

Figure 5. Transgene of MCM6-mCherry, MCM4-Venus, CDC6-mCherry, CDC45-mCherry rescued temperature sensitivity of the corresponding mutant. (A) Indicated strains were grown and serially diluted 5fold on TAP plates. The plates were incubated at 21 or $33{ }^{\circ} \mathrm{C}$ for $5-7$ days. The strain number in red was used in the following experiments. (B) Protein expression of MCM6-mCherry, MCM4-Venus, CDC6-mCherry, ORC1-Venus and CDC45-mCherry were detected by western blotting using anti-mCherry or anti-GFP antibody. CDKB1-mCherry is shown as a positive control. 


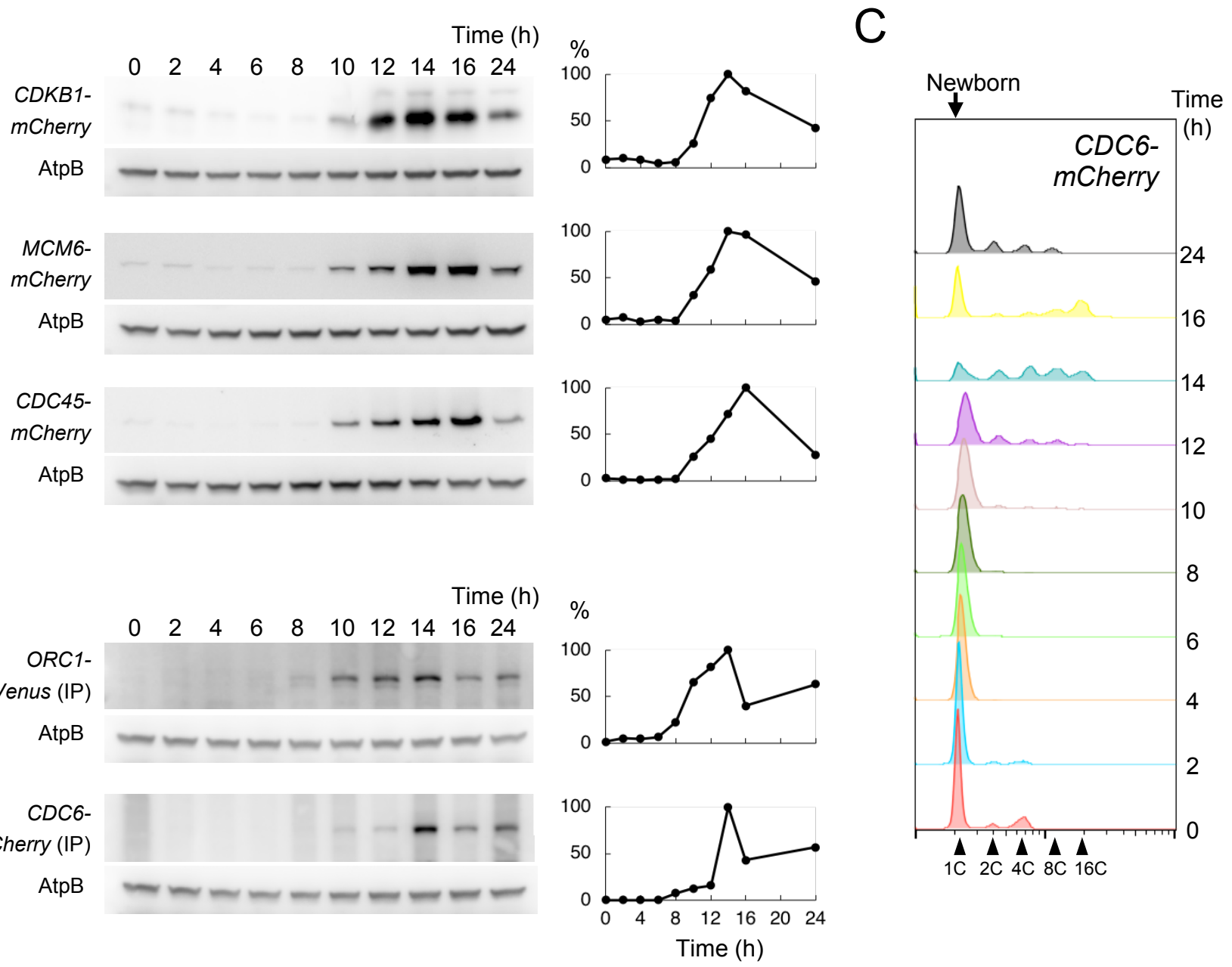

Figure 6. DNA replication proteins are expressed during cell division. (A) CDKB-mCherry, MCM6-mCherry or CDC45-mCherry cells were synchronized in G1 by nitrogen starvation. The cell cycle was released and cells were collected at indicated time points. Protein was extracted and subjected to western blotting analysis. The signal was detected by anti-mCherry antibody. AtpB was used as a loading control. The relative protein expression quantified by ImageJ is shown on the right. The highest expression during the cell cycle was set as $100 \%$. (B) ORC1-Venus or CDC6$m$ Cherry cells was prepared as described in A. Protein was pulled down by GFP or mCherry nanobody coupled with DynaBeads. The signal was detected by anti-GFP or anti-mCherry antibody. AtpB was used as a loading control. The relative protein expression is shown in the right. The highest expression during the cell cycle was set as $100 \%$. (C) The CDC6-mCherry samples used in B were fixed, stained and subjected to FACS analysis to monitor cell cycle progression. 


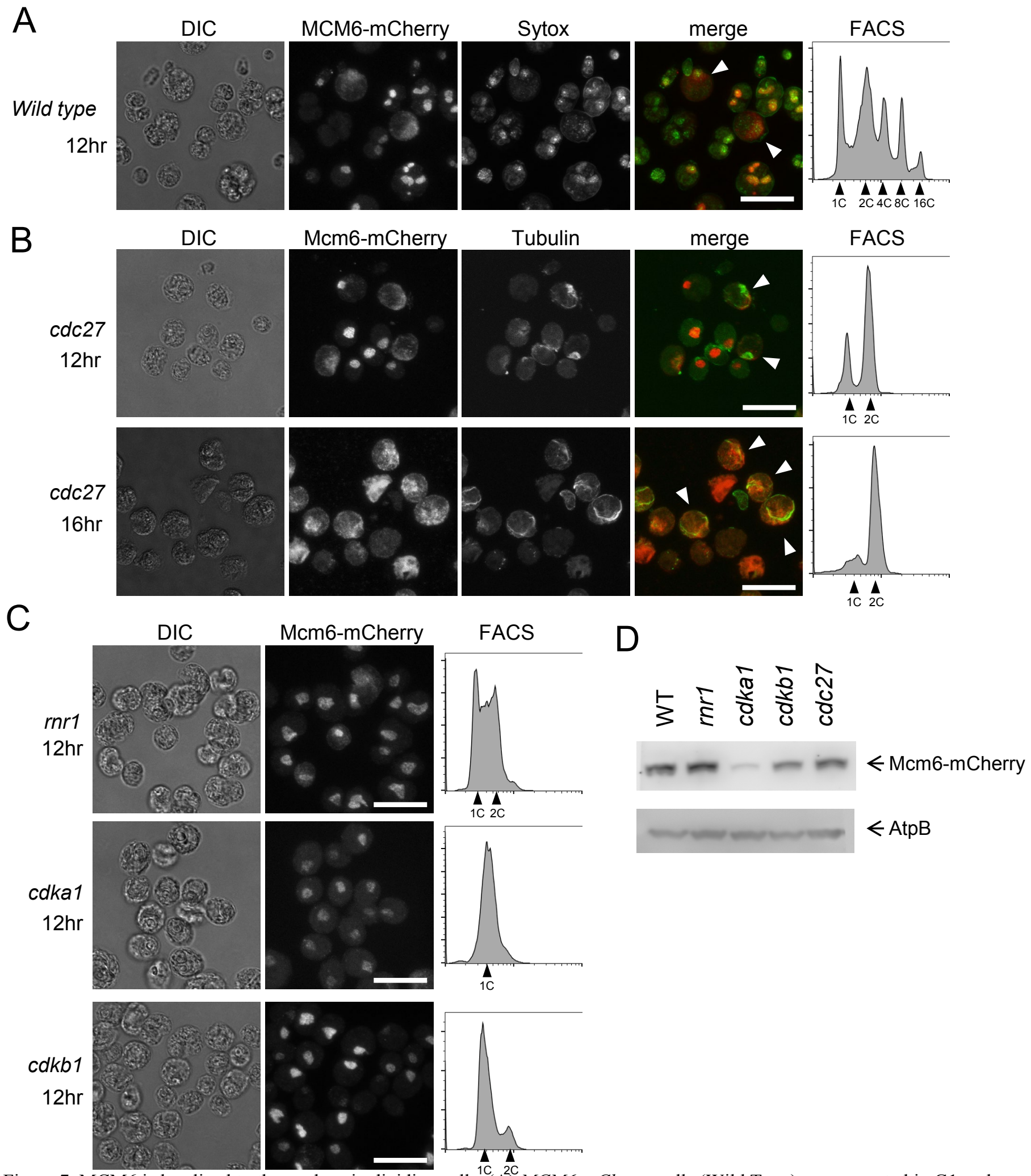

Figure 7. MCM6 is localized to the nucleus in dividing cells. (A) MCM6-mCherry cells (Wild Type) were arrested in G1 and released at $33{ }^{\circ} \mathrm{C}$ for $12 \mathrm{hrs}$. Cells were fixed and stained with anti-mCherry antibody (Red) and Sytox green (Green). White triangles indicate cells with diffused Mcm6. Images were acquired by Confocal microscope. Bars $=20 \mu \mathrm{m}$. The DNA content was analyzed by FACS (right panel) (B) MCM6-mCherry cdc27 cells were collected after $12 \mathrm{hrs}$ or $16 \mathrm{hrs}$ from release and prepared as described in A. White triangles show cells with mitotic spindles. Bars $=20 \mu \mathrm{m}$. The DNA content was analyzed by FACS (right panels). (C) MCM6-mCherry rnr1, MCM6-mCherry cdkal or MCM6-mCherry cdkb1 cells were collected after $12 \mathrm{hrs}$ from the release. Cells were prepared as described in A. Bars $=20 \mu \mathrm{m}$. The DNA content was analyzed by FACS (right panels). (D) Indicated strains were arrested in G1 and collected 12hrs after the release. Proteins were extracted and MCM6 was detected by anti-mCherry antibody. AtpB was used as a loading control. 

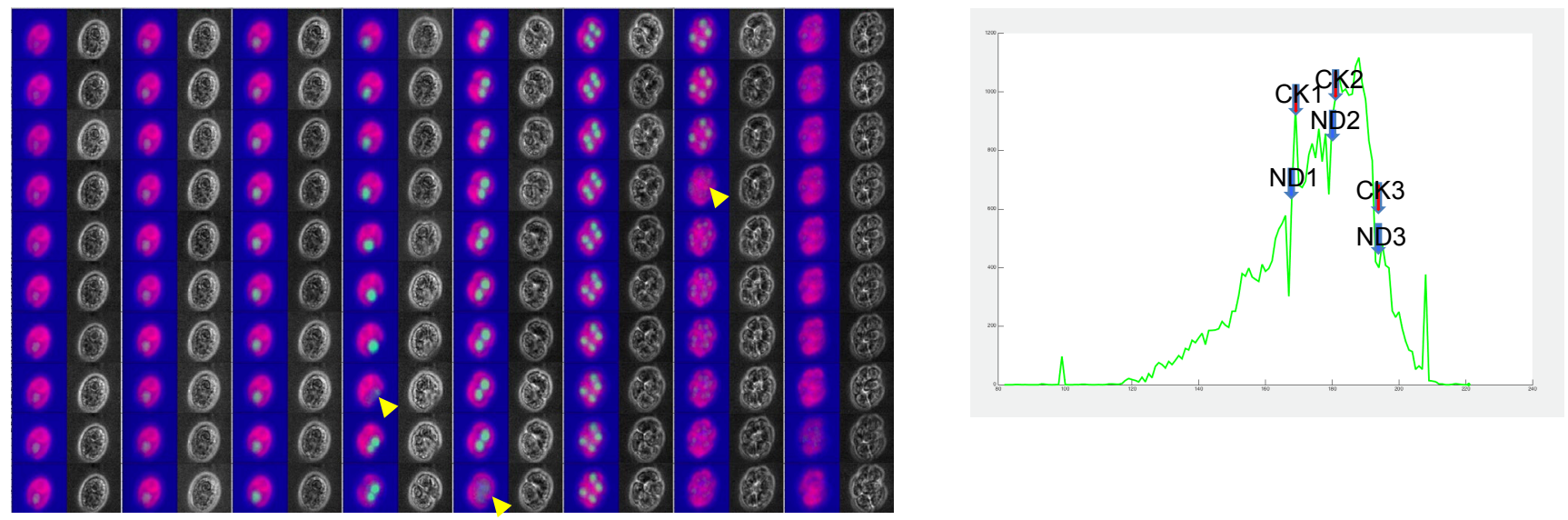

Magenta: Chloroplast, Green: Mcm4-Venus
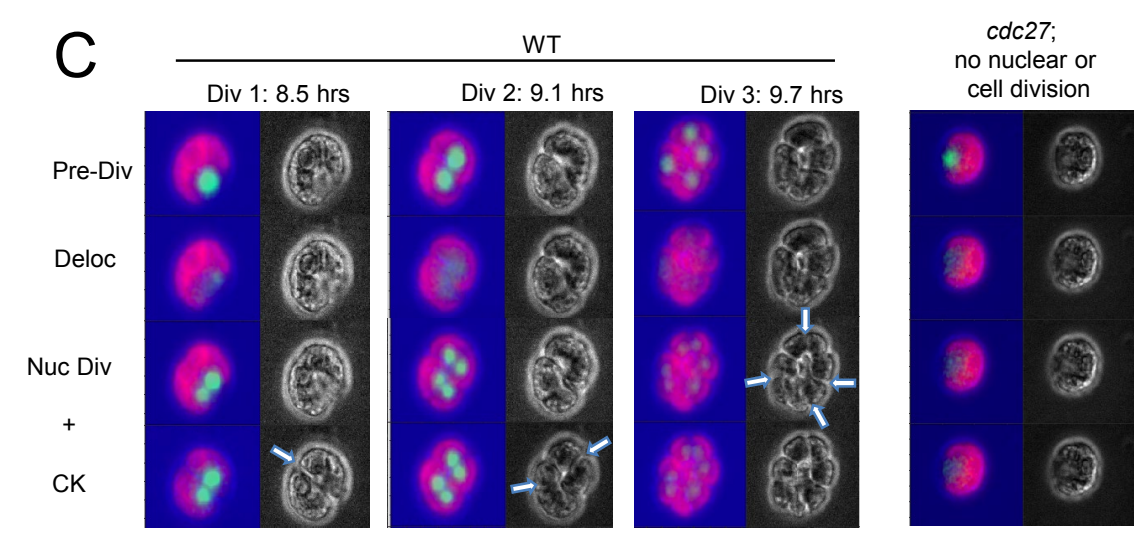

Figure 8. MCM4 is briefly translocated to the cytoplasm in every single S/M phase. (A) MCM4-Venus cells were blocked in G1 by nitrogen starvation and placed on TAP for live imaging by time-lapse microscopy. MCM4-Venus (Green) and chloroplast auto-fluorescence (Magenta). Yellow arrows indicate the time when Mcm4-Venus disappeared from nucleus. (B) The relative intensity of MCM4-Venus signal in (A) was quantified. ND: nuclear division, CK: cytokinesis. (C) The representative images of four stages (Pre-Div: pre division, Deloc: delocalization, Nuc Div: nuclear division, CK: cytokinesis) are shown. Left columns: 1st, 2nd and 3rd division in wild type, right column: 1st division in $c d c 27$. Blue arrows indicate the division site. 
A

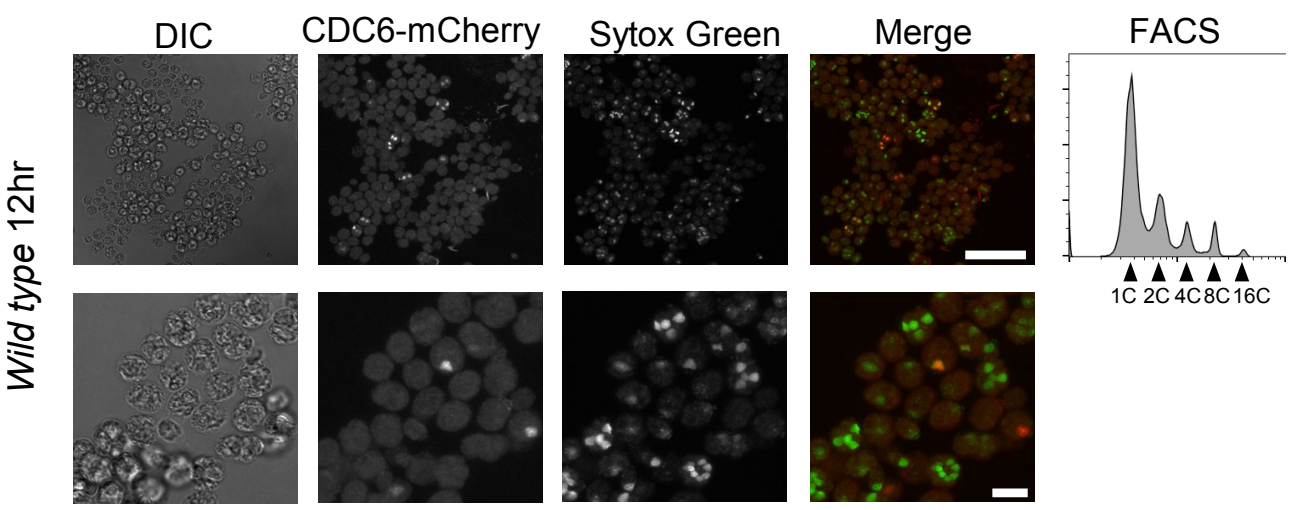

B

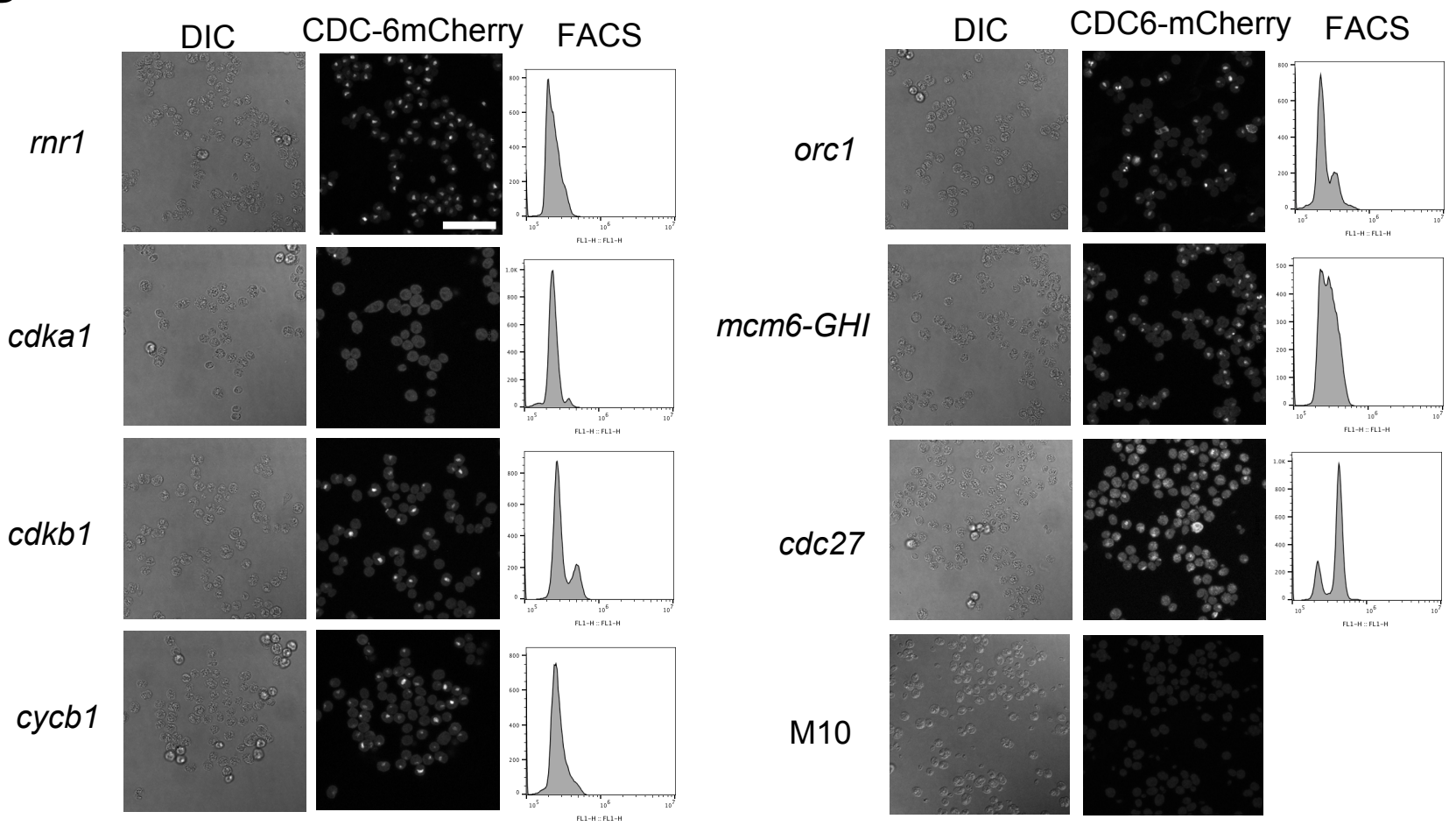

Figure 9. CDC6 protein is transiently localized to the nucleus in dividing cells. (A) CDC6-mCherry (Wild Type) cells were synchronized in G1 and collected 12 hours after the release. Cell were fixed and stained with antimCherry antibody (Red) and Sytox green (Green). Images were acquired by Confocal microscope. Scale bar $=50$ $\mu \mathrm{m}$ (upper row) and $10 \mu \mathrm{m}$ (lower row). DNA content was analyzed by FACS (right panel). (B) CDC6-mCherry in rnrl, cdkal, cdkb1, cycb1, orcl, mcm6-GHI or cdc27 mutant was synchronized in G1 and collected 14 hrs after the release. Cell were fixed and stained with anti-mCherry antibody. Images were acquired by Confocal microscope. DNA content was analyzed by FACS (right panel). M10 strain without CDC6-mCherry was used as a negative control to show background signal. Scale bar $=50 \mu \mathrm{m}$. 


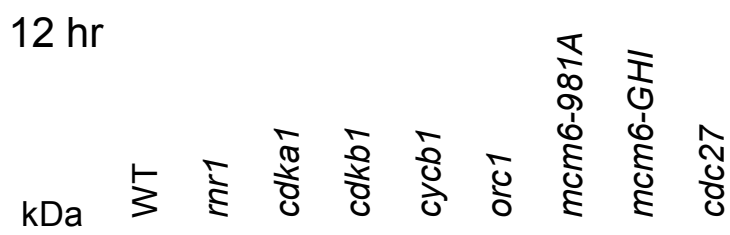

$170-$

$130-$

$72-$
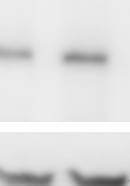
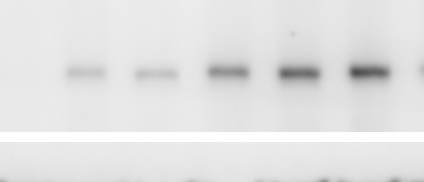

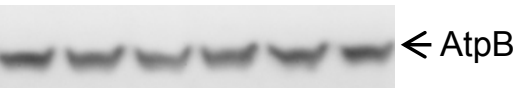

$14 \mathrm{hr}$
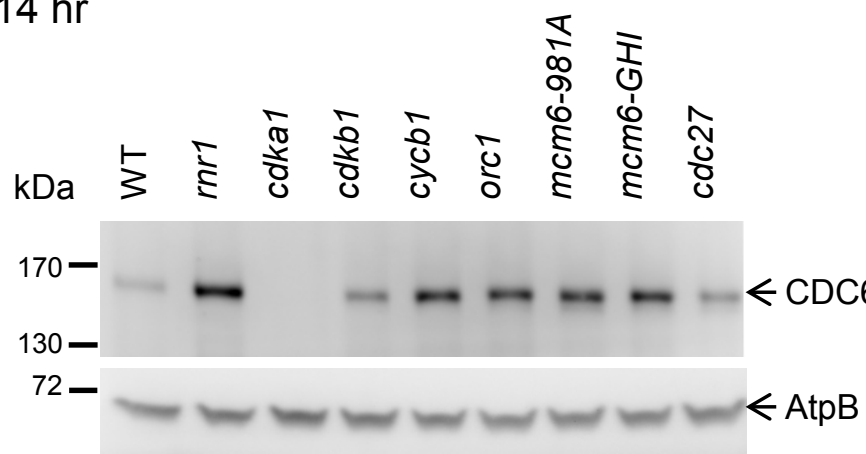

B

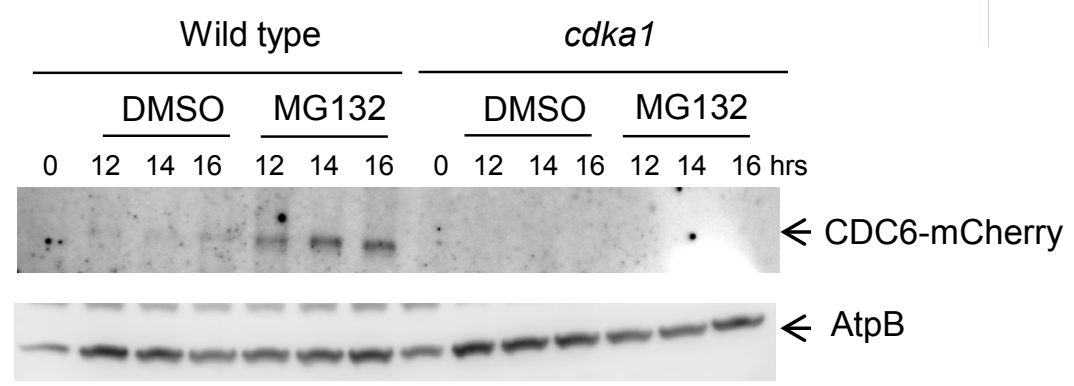

Cdc6-mCherry Western Blot $12 \mathrm{hr}$

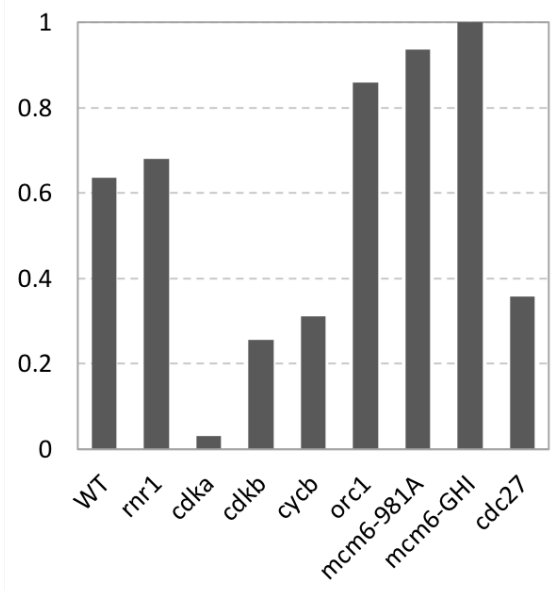

Cdc6-mCherry Western Blot $14 \mathrm{hr}$

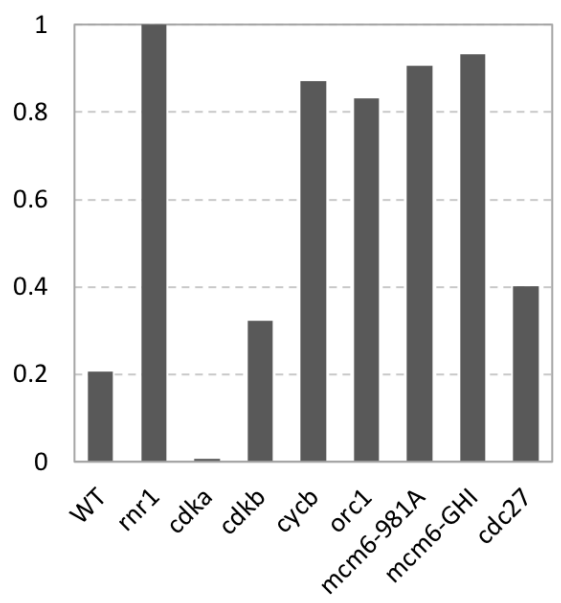

Figure 10. CDC6 protein expression in cell cycle mutants (A) CDC6 protein expression was suppressed in cdkal mutant. Indicated strains were arrested in G1 and collected $12 \mathrm{hrs}$ or $14 \mathrm{hrs}$ after release. Proteins were extracted and CDC6mCherry was pulled down using mCherry nanobody coupled with DynaBeads. The signal was detected by anti-mCherry antibody. AtpB was used as a loading control. The graph shows quantification of relative expression in each strain. (B) CDC6-mCherry (WT) or CDC6-mCherry cdkal (cdkal) cells were arrested in G1 and plated on TAP plates to release the cell cycle arrest. After $10 \mathrm{hrs}$ from release, cells were plated on TAP with DMSO or MG132. Cells were collected 12, 14 or $16 \mathrm{hrs}$ after release corresponding to 2, 4 or 6hrs treatment. Proteins were extracted and CDC6-mCherry was pulled down using mCherry nanobody coupled with DynaBeads. The signal was detected by anti-mCherry antibody. AtpB was used as a loading control. The graph shows quantification of relative expression in each strain. 
A

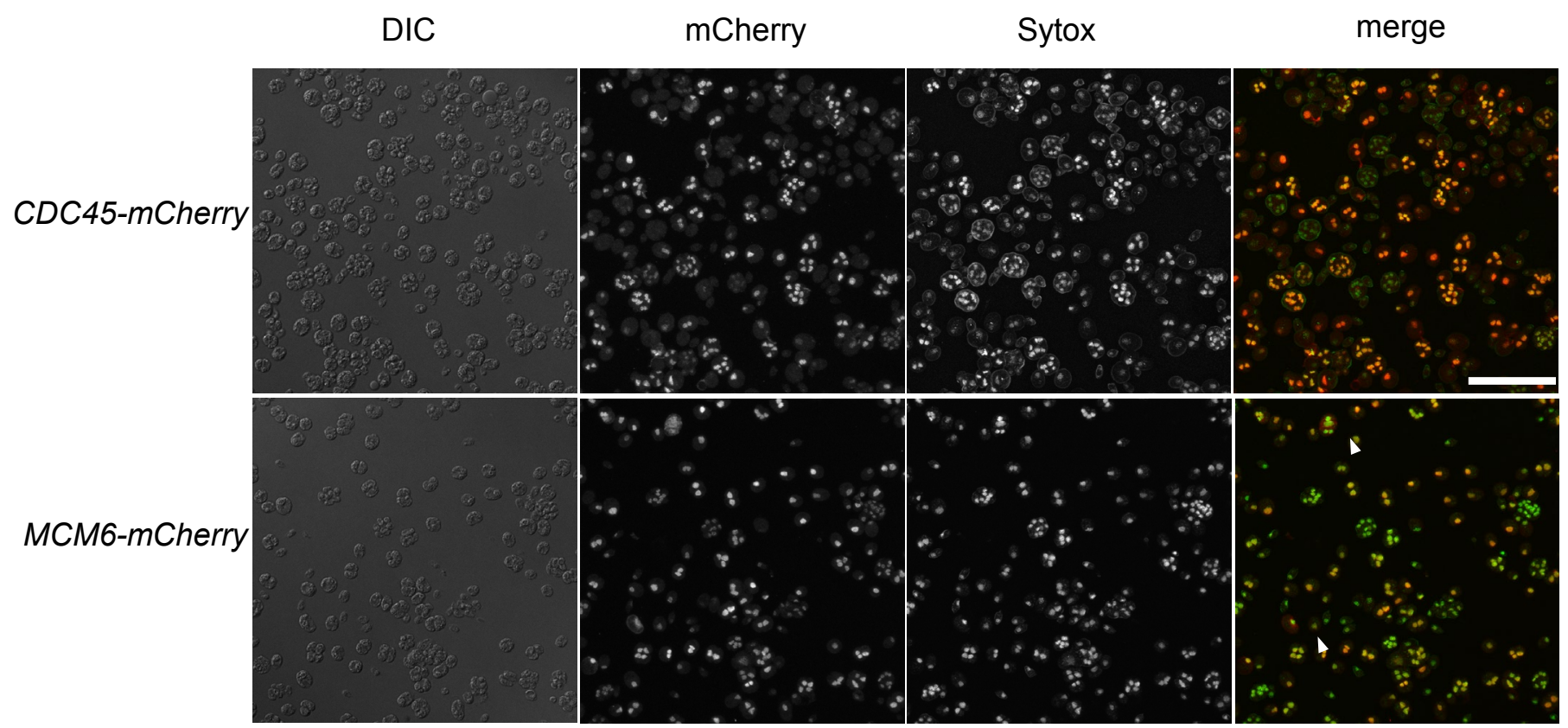

B

DIC

mCherry

Tubulin

merge

MCM6-mCherry cdc27

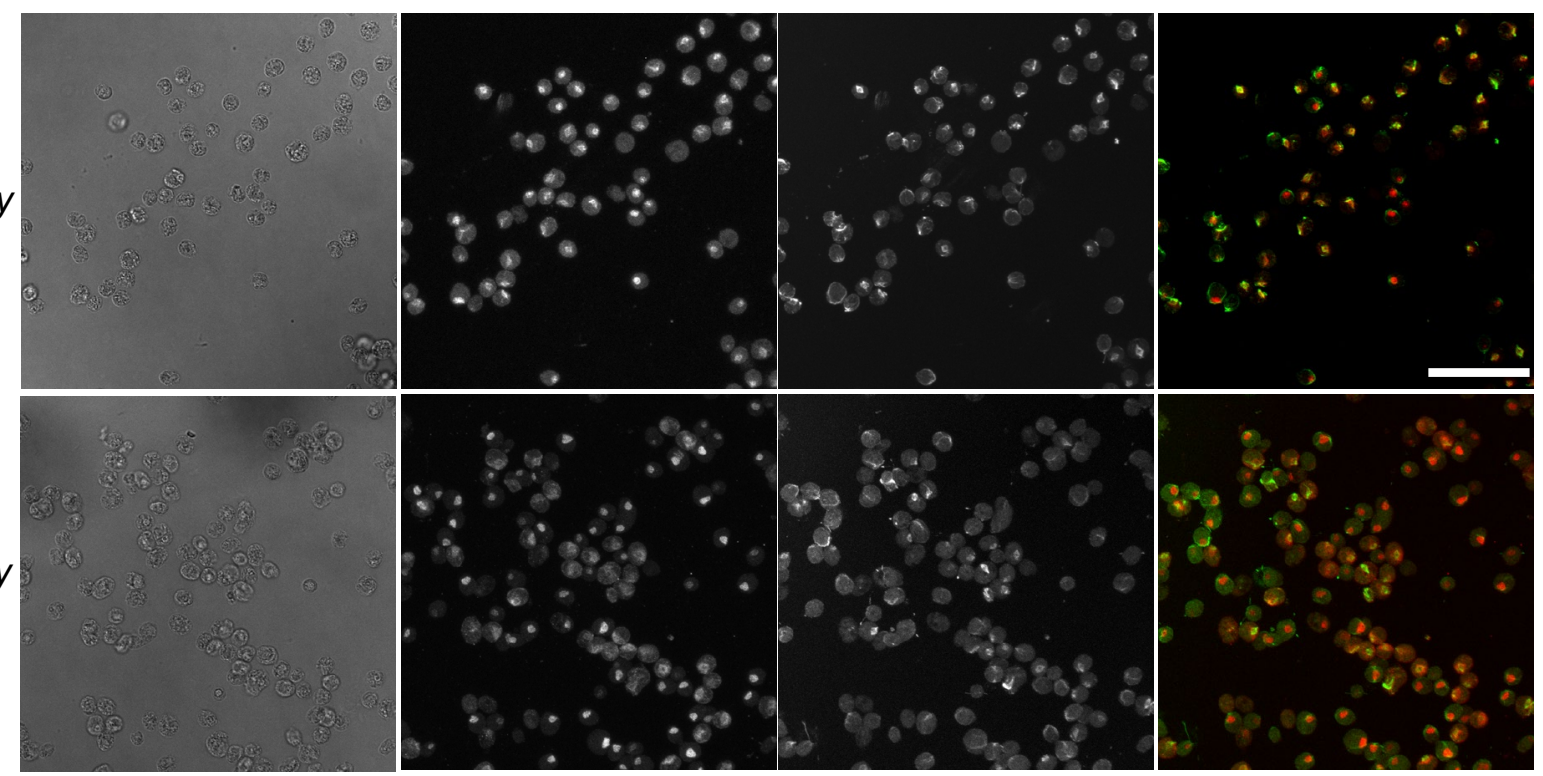

Figure 11. Cdc45 protein is localized to spindles. (A) CDC45-mCherry or MCM6-mCherry cells were synchronized in G1 and collected $12 \mathrm{hrs}$ after release. Cell were fixed and stained with anti-mCherry antibody (Red) and Sytox green (Green). Images were acquired by Confocal microscope. Scale bar $=50 \mu \mathrm{m}$. (B) CDC45-mCherry cdc27 or MCM6-mCherry cdc27 mutant was synchronized in G1 and collected $12 \mathrm{hrs}$ after release. Cell were fixed and stained with anti-mCherry or anti-tubulin antibody. Images were acquired by Confocal microscope. Scale bar $=50 \mu \mathrm{m}$. 